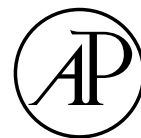

ACADEMIC PRESS
Available online at www.sciencedirect.com

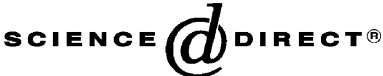

Journal of Computational Physics 186 (2003) 250-278
JOURNAL OF COMPUTATIONAL PHYSICS

www.elsevier.com/locate/jcp

\title{
Coarse-grained stochastic processes and Monte Carlo simulations in lattice systems
}

\author{
Markos A. Katsoulakis ${ }^{\text {a,* }}$, Andrew J. Majda ${ }^{\text {b }}$, Dionisios G. Vlachos ${ }^{\text {c }}$ \\ ${ }^{a}$ Department of Mathematics and Statistics, University of Massachusetts, Amherst, MA 01003, USA \\ ${ }^{\mathrm{b}}$ Courant Institute of Mathematical Sciences and Center for Atmosphere and Ocean Sciences, New York University, \\ New York, NY 10012, USA \\ ${ }^{\mathrm{c}}$ Department of Chemical Engineering and Center for Catalytic Science and Technology, University of Delaware, \\ Newark, DE 19716, USA
}

Received 2 September 2002; received in revised form 15 January 2003; accepted 15 January 2003

\begin{abstract}
In this paper we present a new class of coarse-grained stochastic processes and Monte Carlo simulations, derived directly from microscopic lattice systems and describing mesoscopic length scales. As our primary example, we mainly focus on a microscopic spin-flip model for the adsorption and desorption of molecules between a surface adjacent to a gas phase, although a similar analysis carries over to other processes. The new model can capture large scale structures, while retaining microscopic information on intermolecular forces and particle fluctuations. The requirement of detailed balance is utilized as a systematic design principle to guarantee correct noise fluctuations for the coarse-grained model. We carry out a rigorous asymptotic analysis of the new system using techniques from large deviations and present detailed numerical comparisons of coarse-grained and microscopic Monte Carlo simulations. The coarse-grained stochastic algorithms provide large computational savings without increasing programming complexity or the CPU time per executed event compared to microscopic Monte Carlo simulations.
\end{abstract}

(C) 2003 Elsevier Science B.V. All rights reserved.

Keywords: Hierarchy of Monte Carlo algorithms; Coarse-grained processes and coarse-grained Monte Carlo simulations; Birth-death processes; Detailed balance; Large deviations

\section{Introduction}

Microscopic simulation methods such as molecular dynamics and Monte Carlo algorithms provide a fundamentally derived computational tool capable of describing complex, out-of-equilibrium interactions between atoms and molecules. With the current computing capabilities, these methods yield unprecedented

\footnotetext{
${ }^{*}$ Corresponding author.

E-mail address: markos@math.umass.edu (M.A. Katsoulakis).
} 
insights into numerous problems ranging from physicochemical and biological processes, to pattern recognition and image processing. Despite their widespread use and the substantial progress in related computational methods, molecular simulations are limited to short length and time scales, capable of simulating a relatively small number of atoms/molecules for quite short time periods, while device sizes and morphological features observed in experiments often involve much larger spatial and/or temporal scales. A major obstacle in addressing this multiscale modeling challenge is the lack of a rigorous mathematical and computational framework providing a direct link of microscopic scales to complex mesoscopic and macroscopic phenomena which are dictated by particle/particle interactions.

In this direction, our work focuses on developing a novel stochastic modeling and computational framework, capable of efficiently describing much larger length scales than conventional Monte Carlo (MC) simulations while still incorporating microscopic details. Using as our main paradigm a microscopic spin-flip model for the adsorption and desorption of molecules between a surface and the overlying gas phase, we derive a coarse-grained stochastic jump process describing the system at mesoscopic length scales. The new stochastic process and the associated coarse-grained MC simulations have the potential to capture large scale morphological structures, while they retain microscopic information on intermolecular forces and particle fluctuations. It is numerically shown that the necessary CPU can be reduced by orders of magnitude for large systems and long potentials.

Some aspects of the coarse-grained MC simulations proposed in this paper are in retrospect intuitively clear and have to an extent being partly introduced in a related context in the existing literature. In [20] the authors propose as a computational tool for the stochastic Cahn-Hilliard equation a continuous order parameter MC model. This model is essentially a stochastic phenomenological model, first employed in [17] as a starting point for the derivation of the stochastic Cahn-Hilliard model. In addition, a discrete order parameter stochastic model, similar to the ones derived here from microscopics but without the inclusion of short range interactions, is constructed in [10], and following formal calculations in [17] is used to obtain a stochastic mesoscopic equations for diffusion and adsorption/desorption processes. Furthermore, in the context of a direct derivation of a mesoscopic model from a true microscopic one in the infinite particle limit, the coarse-grained stochastic processes proposed below are also intimately related to intermediate technical steps in the derivation of deterministic and stochastic mesoscopic models arising as mean field limits of Ising systems $[4,7,8,16]$.

The novelty in our approach lies mainly in the following points: First, we derive a hierarchy of successively coarse-grained MC simulations directly from the microscopic processes as approximations in larger length scales and obtain suitable error estimates. These models span a hierarchy of length scales starting from the microscopic to the mesoscopic and macroscopic scales, and involve Markovian birthdeath processes. Detailed asymptotics such as large deviations estimates rigorously justify and clarify these connections.

Second, we study computationally the coarse-grained models, and compare them with microscopic MC simulations, as well as mesoscopic solutions. We demonstrate that the full hierarchy satisfies detailed balance relations and as a result yields self-consistent random fluctuation mechanisms, i.e., consistent mesoscopic models for stochastic noise. Asymptotic analysis and simulations highlight the regimes where microscopic and mesoscopic noise mechanisms are asymptotically identical.

Finally, the new simulations provide significantly faster MC simulations which are easy to implement and are directly related to the microscopics. They also give rise to a new efficient particle method to simulate stochastic PDE (SPDE) derived from microscopic models $[8,10,24]$. The advantages are that we (a) bypass difficulties arising in the simulation of highly singular white noise terms typically arising in SPDE and (b) preserve detail balance at the level of the numerical scheme. We note that in stochastic mesoscopic PDE models with non-conservative mechanisms such as adsorption/desorption it is not clear what is the exact form of the stochastic correction which will ensure detailed balance. 
Concluding the introduction we outline the structure of this paper. In Section 2 we present the microscopic model and set our notation and assumptions. In Section 3 we derive the coarse-grained process and corresponding MC simulations by coarse-graining the interparticle potential and the spin-flip rates in a fashion similar to block spin renormalization [15,9]. In Section 4 we demonstrate that the coarse-grained process satisfies detailed balance, while a Large Deviation analysis provides a mathematically rigorous justification that noise terms are appropriately simulated at the mesoscopic level. In Section 5 we discuss numerical comparisons of microscopic and coarse-grained MC simulations. Finally in Appendix A we outline how the coarse-graining procedure presented here for a spin-flip mechanism can be used in other microscopic processes such as surface diffusion.

\section{Microscopic processes}

We consider as the physical domain the $d$-dimensional torus

$$
T_{d}:=[0,1]^{d},
$$

with the usual periodic boundary conditions. We first divide $T_{d}$ in $m^{d}$ square coarse cells each with volume $1 / m^{d}$. Subsequently each coarse cell is subdivided into $q^{d}$ microcells (or just "cells" for brevity) each with volume $1 /(m q)^{d}$. Thus the torus $T_{d}$ is divided in $N=(m q)^{d}$ cells. Each coarse cell is denoted by

$$
D_{k}=D_{m, k}, \quad k=1, \ldots, m^{d} .
$$

We may now define the coarse lattice corresponding to the coarse cell partition,

$$
\mathscr{L}_{\mathrm{c}}:=\frac{1}{m} \mathbb{Z}^{d} \cap T_{d}
$$

With a slight abuse of notation and suitable ordering, we will consider the integers $k=1, \ldots, m^{d}$ as the lattice points of $\mathscr{L}_{\mathrm{c}}$. The coarse-grained processes defined later are set precisely on this lattice. Furthermore, we define the fine lattice $\mathscr{L}$ corresponding to the microcell partition,

$$
\mathscr{L}:=\frac{1}{m q} \mathbb{Z}^{d} \cap T_{d}
$$

We use the notation $x \in \mathscr{L}$ to denote lattice points of $\mathscr{L}$. The microscopic processes are defined on the finer lattice $\mathscr{L}$. In order to simplify algebraic calculations it is convenient to pick $m$ and $q$ to be powers of 2 but here we choose not to do so hopefully keeping the notation simpler. Finally we point out that from the construction of the lattices we have

$$
\mathscr{L}_{\mathrm{c}} \subset \mathscr{L} .
$$

For simplicity in exposition and convenience in notation we concentrate on the discussion of one-dimensional models, however our results extend to the $d$-dimensional case in a straightforward manner.

\subsection{Microscopic interactions and Gibbs states on $\mathscr{L}$}

Here we consider Ising-type models defined on $\mathscr{L}$. At each lattice site $x \in \mathscr{L}$ an order parameter is allowed to take the values 0 and 1 describing vacant and occupied sites, respectively. In accordance to the classical Ising model, we refer to the order parameter as spin. A spin configuration $\sigma$ is an element of the 
configuration space $\Sigma=\{0,1\}^{\mathscr{L}}$; we write $\sigma=\{\sigma(x): x \in \mathscr{L}\}$, where $\sigma(x) \in\{0,1\}$ denotes the spin at $x$. The energy $H$ of the system, evaluated at $\sigma$, is given by the Hamiltonian,

$$
H(\sigma)=-\frac{1}{2} \sum_{x \in \mathscr{L}} \sum_{y \neq x} J(x-y) \sigma(x) \sigma(y)+\sum h \sigma(x),
$$

where $h$ is the external field and $J$ is the interparticle potential.

Equilibrium states of the Ising model are described by the Gibbs states at the prescribed temperature $T$,

$$
\mu_{\mathscr{L}, \beta}(\mathrm{d} \sigma)=\frac{1}{Z_{\mathscr{L}}} \exp (-\beta H(\sigma)) P_{N}(\mathrm{~d} \sigma) .
$$

Here $P_{N}(\mathrm{~d} \sigma)$ denotes the (product) prior distribution on $\mathscr{L}$ :

$$
P_{N}(\mathrm{~d} \sigma)=\prod_{x \in \mathscr{L}} \rho(\mathrm{d} \sigma(x)),
$$

where

$$
\rho(\sigma(x)=0)=\frac{1}{2}, \quad \rho(\sigma(x)=1)=\frac{1}{2}
$$

is the distribution of a Bernoulli random variable for each $x \in \mathscr{L}$. Finally $\beta=1 /(k T), k$ being the Boltzmann constant and $Z_{\mathscr{L}}$ is the partition function, i.e., a normalizing constant so that $\mu_{\mathscr{L}, \beta}$ is a probability measure defined on the configuration space $\Sigma=\{0,1\}^{\mathscr{L}}$.

The interparticle potentials considered in the sequel are defined on the fine lattice $\mathscr{L}$. We consider potentials with: (a) long range interactions such as electrostatic potentials that extend over the entire lattice and (b) finite range interactions with a given potential length. In the former case each lattice site interacts with all $N-1$ remaining sites on $\mathscr{L}$. In the latter we denote by the integer $2 L$ the total number of interacting

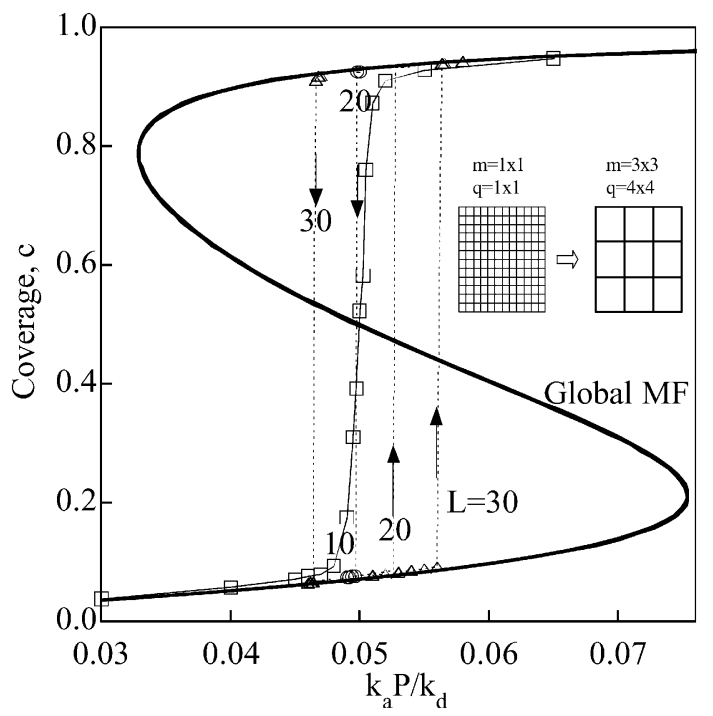

Fig. 1. Comparison of global MF and microscopic MC simulations for various microscopic potential lengths $L$ indicated and $\beta J_{0}=6$. Each point of the isotherm is obtained by starting from the final microconfiguration of the previous isotherm point and performing ensemble averaging over $2 \times 10^{4}$ MCS after $10^{4}$ MCS have been disregarded for equilibration. Hysteresis is observed in $1 \mathrm{D}$ for sufficiently long potentials. For these simulations $m=240$ and $q=1$. The inset is a $2 \mathrm{D}$ schematic of coarse-graining for $N=12 \times 12$. 
neighboring sites of a given point on the one-dimensional lattice $\mathscr{L}$ (see inset in Fig. 1); since $1 / N$ is the lattice size and the potentials are symmetric, the actual potential radius on $\mathscr{L}$ is $L / N$. Since we consider periodic boundary conditions on $\mathscr{L}$, then for $2 L+1=N$ we recover the case of long range interactions. Consequently in both such cases the (symmetric) interaction potential $J=J(x-y)$ can be written as

$$
J(x-y)=\frac{1}{2 L+1} V\left(\frac{N(x-y)}{2 L+1}\right), \quad x, y \in \mathscr{L},
$$

where $V: \mathbf{R} \mapsto \mathbf{R}$ and $V(r)=V(-r), r \in \mathbf{R}$. In the case of finite range interactions we will additionally assume that $V(r)=0,|r| \geqslant 1$. In our numerical experiments in Section 5 we use either a piecewise constant or a Morse-type potential. In the approximation analysis of Section 3, we assume that

$$
V: \mathbf{R} \mapsto \mathbf{R} \text { is smooth in } \mathbf{R} /\{0\}
$$

as well as a suitable blow-up condition at 0 . Note that for $V \in L^{1}(\mathbf{R})$, the choice of the scaling factor $1 / 2 L+1$ in (2.4) implies the summability of the potential $J$, even when $N, L \rightarrow \infty$.

\subsection{Microscopic dynamics}

The dynamics of Ising-type models considered in the literature consists of a sequence of flips and/or spin exchanges that correspond to different physical processes. Here we focus on spin-flip mechanisms and briefly discuss spin exchange mechanisms in Appendix A. More specifically a spin flip at the site $x \in \mathscr{L}$ is a spontaneous change in the order parameter, 1 is converted to 0 and vice versa. Physically this mechanism may describe the desorption of a particle from a surface described by the lattice to the gas phase above and conversely the adsorption of a particle from the gas phase to the surface. Similarly it can describe phase transitions without order parameter conservation.

If $\sigma$ denotes the configuration prior to a flip at $x$, then after the flip the configuration is denoted by $\sigma^{x}$, where

$$
\sigma^{x}(y)= \begin{cases}1-\sigma(x) & \text { when } y=x \\ \sigma(y) & \text { when } y \neq x\end{cases}
$$

We assume that a flip occurs at $x$, when the configuration is $\sigma$, with a rate $c(x, \sigma)$, i.e., a spin flip occurs at $x$, during $[t, t+\Delta t]$ with probability $c(x, \sigma) \Delta t+\mathrm{O}\left(\Delta t^{2}\right)$. Rigorously the underlying stochastic process $\left\{\sigma_{t}\right\}_{t \geqslant 0}$ is defined as a continuous time jump Markov process on $L^{\infty}(\Sigma ; \mathbf{R})$ with generator given by [18]:

$$
L_{N} f(\sigma)=\sum_{x \in \mathbb{Z}^{N}} c(x, \sigma)\left[f\left(\sigma^{x}\right)-f(\sigma)\right], \quad f \in L^{\infty}(\Sigma ; \mathbf{R}) .
$$

An obvious requirement on the resulting dynamics is that they should leave the Gibbs measure (2.2) invariant. This condition is called a detailed balance law, and is equivalent to

$$
c(x, \sigma)=c\left(x, \sigma^{x}\right) \exp \left(-\beta \Delta_{x} H(\sigma)\right) .
$$

The energy difference after performing a spin flip at the site $x$ is

$$
\Delta_{x} H(\sigma)=H\left(\sigma^{x}\right)-H(\sigma)=(2 \sigma(x)-1) U(x),
$$

where

$$
U(x)=\sum_{z \neq x, z \in \mathscr{L}} J(x-z) \sigma(z)-h
$$


is the total energy contribution from the particle interactions with the particle located at the site $x \in \mathscr{L}$, as well as the external field $h$. The simplest type of dynamics satisfying the detailed balance condition is the Metropolis-type dynamics where,

$$
c(x, \sigma)=\Psi\left(-\beta \Delta_{x} H(\sigma)\right)
$$

yielding the relation on $\Psi, \Psi(r)=\Psi(-r) \mathrm{e}^{-r}, r \in \mathbf{R}$. Typical choices of $\Psi$ s are $\Psi(r)=\left(1+\mathrm{e}^{r}\right)^{-1}$ (Glauber dynamics), $\Psi(r)=\mathrm{e}^{-r / 2}$ or $\Psi(r)=\mathrm{e}^{-r^{+}}$(Metropolis dynamics). For such dynamics, the energy barrier for desorption depends only on the energy difference between the initial and final states.

Here we mainly focus on Arrhenius-type dynamics where the activation energy of surface desorption is the energy barrier a particle has to overcome in jumping from the surface to the gas phase. The Arrhenius adsorption/desorption (spin flip) rate is given by

$$
c(x, \sigma)=d_{0}(1-\sigma(x))+d_{0} \sigma(x) \exp \left[-\beta\left(U_{0}+U(x)\right)\right],
$$

where $U(x)$ is defined in (2.5) and $U_{0}$ is the energy associated with the surface binding of the particle at $x$; finally $d_{0}$ is a rate constant that mathematically can be chosen arbitrarily but is physically related to the preexponential of the microscopic processes.

\section{Coarse-graining of microscopic processes}

In this section our goal is to derive an approximate mesoscopic Markov process for a suitably defined coarse-grained variable $\eta_{t}$ defined on the coarse lattice $\mathscr{L}_{\mathrm{c}}$ by equivalently obtaining the corresponding semigroup generator, starting from the microscopic process $\sigma_{t}$.

We first introduce the coarse-grained random process defined as an average of the microscopic process $\left\{\sigma_{t}\right\}_{t \geqslant 0}$ over each coarse cell $D_{k}$ :

$$
\eta_{t}(k)=\sum_{y \in D_{k}} \sigma_{t}(y), \quad k=1, \ldots, m
$$

The random process $\eta_{t}(k)$ is defined for each coarse cell $D_{k}$ of the lattice $\mathscr{L}_{\mathrm{c}}$ and satisfies the constraint $0 \leqslant \eta_{t}(k) \leqslant q$, since each coarse cell contains $q$ microcells. Equivalently we may also consider the averaged version (termed below as coverage)

$$
\bar{\eta}_{t}(k)=\frac{1}{q} \sum_{y \in D_{k}} \sigma_{t}(y)=q^{-1} \eta_{t}(k) .
$$

First recall from the definition of the generator of $\left\{\sigma_{t}\right\}_{t \geqslant 0}$ that for all test functions $f \in L^{\infty}(\Sigma ; \mathbf{R})$,

$$
\frac{\mathrm{d}}{\mathrm{d} t} E f\left(\sigma_{t}\right)=E L_{N} f(\sigma)=E \sum_{x \in \mathscr{L}} c\left(x, \sigma_{t}\right)\left[f\left(\sigma_{t}^{x}\right)-f\left(\sigma_{t}\right)\right] .
$$

From now on and unless it is otherwise necessary we suppress the $t$-dependence of $\sigma_{t}$ or $\eta_{t}$.

Motivated from the definition of $\eta$ we define the new configuration space

$$
\mathscr{H}_{m, q}=\{0,1, \ldots, q\}^{\mathscr{L}_{\mathrm{c}}},
$$

where $\eta=\left\{\eta(k): k \in \mathscr{L}_{\mathrm{c}}\right\}$ and $\eta(k) \in\{0,1, \ldots, q\}$ is the coverage of the coarse cell $D_{k}$. We also define the mapping $F: \Sigma \mapsto \mathscr{H}_{m, q}$, where 


$$
F(\sigma)(k):=\sum_{y \in D_{k}} \sigma(y)=\eta(k), \quad k=1, \ldots, m .
$$

Then for any test function $g \in L^{\infty}\left(\mathscr{H}_{m, q} ; \mathbf{R}\right)$ we have that

$$
f(\sigma):=g(F(\sigma))=g(\eta)
$$

is a test function in $L^{\infty}(\Sigma ; \mathbf{R})$ and therefore from (3.1) we have:

$$
\frac{\mathrm{d}}{\mathrm{d} t} E f(\sigma)=E \sum_{x \in \mathscr{L}} c(x, \sigma)\left[f\left(\sigma^{x}\right)-f(\sigma)\right]=E \sum_{k \in \mathscr{L}_{\mathrm{c}}} \sum_{x \in D_{k}} c(x, \sigma)\left[f\left(\sigma^{x}\right)-f(\sigma)\right] .
$$

For $x \in D_{k}$ we have

$$
F\left(\sigma^{x}\right)(k)=\sum_{y \in D_{k}} \sigma^{x}(y)= \begin{cases}\eta(k)+1 & \text { when } \sigma(x)=0, \\ \eta(k)-1 & \text { when } \sigma(x)=1,\end{cases}
$$

thus, we obtain from (3.2) that

$$
f\left(\sigma^{x}\right)-f(\sigma)=(1-\sigma(x))\left[g\left(\eta+\delta_{k}\right)-g(\eta)\right]+\sigma(x)\left[g\left(\eta-\delta_{k}\right)-g(\eta)\right], \quad x \in D_{k} .
$$

Here $\delta_{k} \in \mathscr{H}_{m, q}$ is the configuration with a single particle at the site $k \in \mathscr{L}_{\mathrm{c}}$. Replacing in (3.3) we have for any test function $g \in L^{\infty}\left(\mathscr{H}_{m, q} ; \mathbf{R}\right)$ :

$$
\begin{aligned}
\frac{\mathrm{d}}{\mathrm{d} t} E g(\eta) & =\frac{\mathrm{d}}{\mathrm{d} t} E f(\sigma)=E \sum_{x \in \mathscr{L}} c(x, \sigma)\left[f\left(\sigma^{x}\right)-f(\sigma)\right] \\
& =E \sum_{k \in \mathscr{L}_{c}} \sum_{x \in D_{k}} c(x, \sigma)(1-\sigma(x))\left[g\left(\eta+\delta_{k}\right)-g(\eta)\right]+c(x, \sigma) \sigma(x)\left[g\left(\eta-\delta_{k}\right)-g(\eta)\right] .
\end{aligned}
$$

Using (2.6), and that $\sigma(x) \in\{0,1\}$, we obtain

$$
\sum_{x \in D_{k}} c(x, \sigma)(1-\sigma(x))=\sum_{x \in D_{k}} d_{0}(1-\sigma(x))=d_{0}(q-\eta(k))
$$

and

$$
\sum_{x \in D_{k}} c(x, \sigma) \sigma(x)=\sum_{x \in D_{k}} d_{0} \sigma(x) \exp \left[-\beta\left(U_{0}+U(x)\right)\right] .
$$

Relation (3.5) clearly depends only on $\eta$ and according to (3.4) yields the rate with which the value $\eta(k)$ is increased by 1 , i.e., the adsorption of a single particle occurs in the coarse cell $D_{k}$. Thus we define the coarsegrained adsorption rate

$$
c_{\mathrm{a}}(k, \eta):=d_{0}[q-\eta(k)] .
$$

Similarly (3.6) corresponds to the rate with which the value $\eta(k)$ is decreased by 1, i.e., the desorption of a single particle occurs in the coarse cell $D_{k}$. If, as in (3.5), we can write (3.6) only as a function $c_{d}(k, \eta)$ of the coarse-grained variable $\eta$ (without explicit dependence on $\sigma$ ), then the right-hand side of (3.4) will yield a coarse-grained Markov process generator of birth-death type for the process $\eta$ :

$$
L_{\mathrm{c}} g(\eta)=\sum_{k \in \mathscr{L}_{\mathrm{c}}} c_{\mathrm{a}}(k, \eta)\left[g\left(\eta+\delta_{k}\right)-g(\eta)\right]+c_{\mathrm{d}}(k, \eta)\left[g\left(\eta-\delta_{k}\right)-g(\eta)\right] .
$$


Thus the resulting coarse-grained process $\eta$ will be a Markov process and will provide a stochastic mesoscopic model for the microscopic adsorption/desorption mechanisms. Below we concentrate on the last missing piece in this attempt which is the (approximate) derivation from (3.6) of a mesoscopic desorption rate $c_{\mathrm{d}}(k, \eta)$.

\subsection{Coarse-grained potential and desorption rate}

We recall from (2.5) that $U(x)=\sum_{z \neq x, z \in \mathscr{L}} J(x-z) \sigma(z)-h$. For a fixed $x \in \mathscr{L}$, we have that $x \in D_{l}$ for some $l \in \mathscr{L}_{\mathrm{c}}$ and we rewrite the summation in the formula for $U$ as

$$
\begin{aligned}
\sum_{z \neq x, z \in \mathscr{L}} J(x-z) \sigma(z) & =\sum_{k \in \mathscr{L}_{\mathrm{c}}} \sum_{z \neq x, z \in D_{k}} J(x-z) \sigma(z) \\
& =\sum_{z \neq x, z \in D_{l}} J(x-z) \sigma(z)+\sum_{k \neq l, k \in \mathscr{L}_{\mathrm{c}}} \sum_{z \in D_{k}} J(x-z) \sigma(z) \\
& :=\mathrm{I}+\mathrm{II} .
\end{aligned}
$$

The term II consists of the long range interactions of $x \in D_{l}$ with particles in other coarse cells $D_{k}, k \neq l$, while the term I gathers interactions within the cell $D_{l}$.

We now define the coarse-grained potential by including all contributions of pairwise microscopic interactions between coarse cells and within the same coarse cell, averaged over the subregion of $T_{1} \times T_{1}$ corresponding to $D_{l} \times D_{k}$, denoted by a slight abuse of notation also as $D_{l} \times D_{k}$. More specifically we set

$$
\bar{J}(k, l)=m^{2} \iint_{D_{l} \times D_{k}} J(r-s) \mathrm{d} r \mathrm{~d} s
$$

where the area of $D_{l} \times D_{k}$ is equal to $1 / \mathrm{m}^{2}$. Similarly we define

$$
\bar{V}(k, l)=m^{2} \iint_{D_{l} \times D_{k}} V\left(\frac{N|r-s|}{2 L+1}\right) \mathrm{d} r \mathrm{~d} s .
$$

Since the potential $J$ is given by $J(x-y)=(1 /(2 L+1)) V(N(x-y) / 2 L+1)$, we have

$$
J(x-y)=J\left(x^{\prime}-y^{\prime}\right)+\frac{1}{2 L+1} \mathrm{O}\left(\frac{N}{(2 L+1) m}\right)=J\left(x^{\prime}-y^{\prime}\right)+\frac{1}{2 L+1} \mathrm{O}\left(\frac{q}{2 L+1}\right),
$$

when $x, x^{\prime} \in D_{l}, y, y^{\prime} \in D_{k}, k \neq l$. Finally we have

$$
J(x-y)=\bar{J}(k, l)+\frac{1}{2 L+1} \mathrm{O}\left(\frac{q}{2 L+1}\right), \quad x \in D_{l}, \quad y \in D_{k},
$$

where

$$
\mathrm{O}\left(\frac{q}{2 L+1}\right) \leqslant \frac{q}{2 L+1} \sup _{\substack{x \in D_{l} \\ y \in D_{k}}}\left(\left|\partial_{x} V\right|+\left|\partial_{y} V\right|\right), \quad k \neq l
$$


Now using (3.10) we can rewrite II in terms of the coarse-grained variable

$$
\begin{aligned}
\mathrm{II} & =\sum_{\substack{k \in \mathscr{L}_{\mathrm{c}} \\
k \neq l}} \sum_{\substack{z \in D_{k} \\
|z-x| \leqslant L / N}} J(x-z) \sigma(z)=\sum_{\substack{k \in \mathscr{L}_{\mathrm{c}} \\
k \neq l^{\mathrm{c}}}}\left(\bar{J}(k, l)+\frac{1}{2 L+1} \mathrm{O}\left(\frac{q}{2 L+1}\right)\right) \sum_{\substack{z \in D_{k} \\
|z-x| \leqslant L / N}} \sigma(z) \\
& =\sum_{\substack{k \in \mathscr{L}_{\mathrm{c}} \\
k \neq l}} \bar{J}(k, l) \eta(k)+\frac{1}{2 L+1} \mathrm{O}\left(\frac{q}{2 L+1}\right) \sum_{\substack{z \in \mathscr{L} \\
z-x \mid \leqslant L / N}} \sigma(z)=\sum_{\substack{k \in \mathscr{L}_{\mathrm{c}} \\
k \neq l}} \bar{J}(l, k) \eta(k)+\mathrm{O}\left(\frac{q}{2 L+1}\right) .
\end{aligned}
$$

Improved error estimates can be obtained in a straightfoward manner if $V$ decays far from the origin (or $J$ decays away from the diagonal), hence the interactions between $D_{k}$ and $D_{l}$ will accordingly decay.

If the potential $V$ is smooth in $\mathbf{R}$, then the short range interactions included in term I can also be treated similarly: for $x \in D_{l}$,

$$
\begin{aligned}
I & =\sum_{z \neq x, z \in D_{l}} J(x-z) \sigma(z)=\bar{J}(0,0) \sum_{z \neq x, z \in D_{l}} \sigma(z)+\frac{1}{2 L+1} \mathrm{O}\left(\frac{q}{2 L+1}\right) \\
& =\bar{J}(0,0)(\eta(l)-\sigma(x))+\frac{1}{2 L+1} \mathrm{O}\left(\frac{q}{2 L+1}\right) .
\end{aligned}
$$

On the other hand we can also handle potentials with a singularity at the origin, provided the following condition is satisfied:

$$
\iint_{T_{1} \times T_{1}}\left|\partial_{r} J(r-s)\right| \mathrm{d} r \mathrm{~d} s<\infty
$$

In this case we have the $L^{\infty}$ bound in $D_{l} \times D_{l}$

$$
|J(x-y)-\bar{J}(0,0)| \leqslant 2 m \iint_{D_{l} \times D_{l}}\left|\partial_{r} J(r-s)\right| \mathrm{d} r \mathrm{~d} s,
$$

where the right-hand side clearly decays as $m \rightarrow \infty$, since the area of $D_{l} \times D_{l}$ is equal to $1 / \mathrm{m}^{2}$.

Next we complete the derivation of the coarse-grained process, where the errors used are the ones derived in the case of the smooth potential only; singular potentials are handled similarly. We can now write for $x \in D_{l}$,

$$
U(x)=\sum_{\substack{k \in \mathscr{C}_{\mathrm{c}} \\ k \neq l}} \bar{J}(l, k) \eta(k)+\bar{J}(0,0)(\eta(l)-\sigma(x))-h+\mathrm{O}\left(\frac{q}{2 L+1}\right) .
$$

Consequently (3.6) becomes

$$
\begin{aligned}
\sum_{x \in D_{k}} d_{0} \sigma(x) \exp \left[-\beta\left(U_{0}+U(x)\right)\right] & =\exp \left[-\beta\left(U_{0}+\bar{U}(k)+\mathrm{O}\left(\frac{q}{2 L+1}\right)\right)\right] \sum_{x \in D_{k}} c_{0} \sigma(x) \\
& =\exp \left[\mathrm{O}\left(\frac{q}{2 L+1}\right)\right] d_{0} \eta(k) \exp \left[-\beta\left(U_{0}+\bar{U}(k)\right)\right]
\end{aligned}
$$

where we defined

$$
\bar{U}(l)=\sum_{\substack{k \in \mathscr{S}_{\mathrm{c}} \\ k \neq l}} \bar{J}(l, k) \eta(k)+\bar{J}(0,0)(\eta(l)-1)-h .
$$


Note also that

$$
U(x)=\bar{U}(l)+\mathrm{O}\left(\frac{q}{2 L+1}\right) .
$$

By momentarily disregarding the $\mathrm{O}(q / 2 L+1)$ term in (3.11) we now define

$$
c_{\mathrm{d}}(k, \eta)=d_{0} \eta(k) \exp \left[-\beta\left(U_{0}+\bar{U}(k)\right)\right] \text {. }
$$

Using (3.5) and (3.11), relation (3.4) becomes

$$
\frac{\mathrm{d}}{\mathrm{d} t} E g(\eta)=E \sum_{k \in \mathscr{L}_{\mathrm{c}}} c_{\mathrm{a}}(k, \eta)\left[g\left(\eta+\delta_{k}\right)-g(\eta)\right]+\exp [\mathrm{O}(q / 2 L+1)] c_{\mathrm{d}}(k, \eta)\left[g\left(\eta-\delta_{k}\right)-g(\eta)\right]
$$

for any test function $g \in L^{\infty}\left(\mathscr{H}_{m, q} ; \mathbf{R}\right)$.

Relation (3.15) suggests that at least, when $2 L+1 \gg q$, the coarse-grained variable $\eta$ is "approximately" a Markov process with generator given by the right-hand side of (3.15). We can now define a corresponding new process $\eta$ from (3.15) by disregarding the $\mathrm{O}(q / 2 L+1)$ term. Note the similarity of the calculations in (3.8)-(3.12) to the block spin renormalization procedure [9].

In other ad hoc models in the literature [10,17], the interactions within the same cell are ignored; in fact, the term $\mathrm{I}$ is lower order for $2 L+1 \gg q$, but this is not true any more if the potential radius $L$ is relatively short, and/or the interactions are very strong. Here we included same cell interactions so that when we coarse grain beyond the interaction radius $L$, and thus kill all cross-cell interactions, to still obtain the global mean field (MF) theory. Indeed we now obtain a whole "hierarchy" of MC models from nearest neighbor to mean field where the latter does not include interactions but includes noise, unlike the usual ODE mean field theories. The proper addition of interactions within the cell in the coarse model is derived from microscopics; in the end the dynamics still obey detailed balance, as established in the next section, even after adding the interactions within the cell. In Section 5, we numerically show that these fluctuations are particularly important close to turning points.

\subsection{The coarse-grained processes}

We define the birth-death Markov process $\eta$ on the configuration space

$$
\mathscr{H}_{m, q}=\{0,1, \ldots, q\}^{\mathscr{L}_{\mathrm{c}}},
$$

where $\eta=\left\{\eta(k): k \in \mathscr{L}_{\mathrm{c}}\right\}$ and $\eta(k) \in\{0,1, \ldots, q\}$ for the coarse cell $D_{k}, k=1, \ldots, m$. A typical configuration $\eta_{0}$ has the form

$$
\eta_{0}=\left(\lambda_{1}, \lambda_{2}, \ldots, \lambda_{m}\right)
$$

where $\lambda_{i} \in\{0,1, \ldots, q\}$. The generator of the process suggested by (3.15) is

$$
L_{\mathrm{c}} g(\eta)=\sum_{k \in \mathscr{L}_{\mathrm{c}}} c_{\mathrm{a}}(k, \eta)\left[g\left(\eta+\delta_{k}\right)-g(\eta)\right]+c_{\mathrm{d}}(k, \eta)\left[g\left(\eta-\delta_{k}\right)-g(\eta)\right] .
$$

The update rate with which the value $\eta(k)$ is increased by 1, i.e., the adsorption rate of a single particle in the coarse cell $D_{k}$ is

$$
c_{\mathrm{a}}(k, \eta):=d_{0}[q-\eta(k)] .
$$


Similarly the rate with which the value $\eta(k)$ is decreased by 1, i.e., the desorption rate of a single particle in the coarse cell $D_{k}$ is

$$
c_{\mathrm{d}}(k, \eta)=d_{0} \eta(k) \exp \left[-\beta\left(U_{0}+\bar{U}(k)\right)\right]
$$

where $\bar{U}$ is defined in (3.12). Then for any test function $g \in L^{\infty}\left(\mathscr{H}_{m, q} ; \mathbf{R}\right)$ we have

$$
\frac{\mathrm{d}}{\mathrm{d} t} E g(\eta)=E \sum_{k \in \mathscr{L}_{\mathrm{c}}} c_{\mathrm{a}}(k, \eta)\left[g\left(\eta+\delta_{k}\right)-g(\eta)\right]+c_{\mathrm{d}}(k, \eta)\left[g\left(\eta-\delta_{k}\right)-g(\eta)\right]
$$

\subsection{Wavelet-based coarse grainings}

Wavelets with $M$ vanishing moments can be used in the construction of the coarse-grained potential instead of the simple averaging procedure in (3.8). More specifically we consider the compactly supported pair of functions $\phi$ and $\psi$ defined on $(-\infty, \infty)$ with the properties [2],

$$
\int \phi(x) \mathrm{d} x=1, \quad \int \psi(x) x^{l} \mathrm{~d} x=0, \quad l=0,1, \ldots, M-1,
$$

where

$$
\begin{aligned}
& \phi(x)=\sqrt{2} \sum_{k=0}^{2 M-1} h_{k+1} \phi(2 x-k), \\
& \psi(x)=\sqrt{2} \sum_{k=0}^{2 M-1} g_{k+1} \phi(2 x-k)
\end{aligned}
$$

and $g_{k}=(-1)^{k-1} h_{2 M-k-1}, k=1, \ldots, 2 M$. Assuming that $m=2^{j}$, we define corresponding wavelets as the dilations and translations of $\phi$ and $\psi$,

$$
\phi_{k}^{m}(x):=m \phi(m x-k+1), \quad \psi_{k}^{m}(x):=m \psi(m x-k+1), \quad k=1,2, \ldots
$$

When $\phi(x)=\chi_{[0,1)}$ and $\psi=\chi_{[0,5)}-\chi_{[.5,1)}$ we obtain the usual Haar wavelets.

We can now define the coarse-grained potential as

$$
\bar{J}(k, l)=\iint J(r-s) \phi_{k}^{m}(r) \phi_{l}^{m}(s) \mathrm{d} r \mathrm{~d} s .
$$

In the case of the Haar basis, (3.20) is precisely the coarse-grained potential defined in (3.8). Note that the multiplying factor $m$ in the definition of $\phi_{k}^{m}, \psi_{k}^{m}$, instead of the usual $m^{1 / 2}$ guaranteeing the orthonormality of $\psi_{k}^{m}$ in the standard wavelet expansions, is necessary here to ensure quadrature formulae with errors such as (3.10) for the coarse-grained potentials (3.20).

By using the wavelet-based definition (3.20) we gain some notable advantages over (3.8). First the integrals (3.20) can be calculated by the method proposed in [1] to $2 M$ order of accuracy by solving the difference equations satisfied exactly by the matrix $(\bar{J}(k, l))_{k l}$, as well as using the vanishing moment properties of the autocorrelation function of $\phi$. Secondly by using the non-standard form (see [2]) we can systematically compress $(\bar{J}(k, l))_{k l}$ by discarding interactions when $k$ and $l$ are sufficiently apart, i.e., restrict the bandwidth at any given coarse resolution $1 / \mathrm{m}$. 


\section{Detailed balance and large deviations principle}

Here we study dynamic properties of the coarse-grained process and connect with microscopic and mesoscopic theories.

\subsection{Invariant measures and detailed balance}

Here we derive the invariant measure for the coarse-grained process and in particular we show it satisfies the detailed balance condition similarly to the true microscopic dynamics $\left\{\sigma_{t}\right\}$.

First we define the new coarse-grained Hamiltonian corresponding to (3.19). We rewrite (2.1) using (3.13) and the error estimates in Section 3.1 for a constant external field $h$, as

$$
\begin{aligned}
H(\sigma) & =-\frac{1}{2} \sum_{l \in \mathscr{L}_{\mathrm{c}}} \sum_{x \in D_{l}}\left(\sum_{\substack{k \in \mathscr{L}_{\mathrm{c}} \\
k \neq l}} \bar{J}(k, l) \eta(k)+\bar{J}(0,0)(\eta(l)-1)\right) \sigma(x)+\sum_{k \in \mathscr{L}_{\mathrm{c}}} h \eta(k)+\mathrm{O}(N q / 2 L+1) \\
& =-\frac{1}{2} \sum_{l \in \mathscr{L}_{\mathrm{c}}} \sum_{\substack{k \in \mathscr{S}_{\mathrm{c}} \\
k \neq l}} \bar{J}(k, l) \eta(k) \eta(l)-\frac{\bar{J}(0,0)}{2} \sum_{l \in \mathscr{L}_{\mathrm{c}}} \eta(l)(\eta(l)-1)+\sum_{k \in \mathscr{L}_{\mathrm{c}}} h \eta(k)+\mathrm{O}(N q / 2 L+1) .
\end{aligned}
$$

We now define the coarse-grained Hamiltonian

$$
\bar{H}(\eta)=-\frac{1}{2} \sum_{l \in \mathscr{L}_{\mathrm{c}}} \sum_{k \in \mathscr{L}_{\mathrm{c}} k \neq l} \bar{J}(k, l) \eta(k) \eta(l)-\frac{\bar{J}(0,0)}{2} \sum_{l \in \mathscr{L}_{\mathrm{c}}} \eta(l)(\eta(l)-1)+\sum_{k \in \mathscr{L}_{\mathrm{c}}} h \eta(k) .
$$

Note that

$$
H(\sigma)=\bar{H}(F(\sigma))+\mathrm{O}(N q / 2 L+1)
$$

where we have defined $F(\sigma)(k)=\sum_{y \in D_{k}} \sigma(y)=\eta(k)$. In the usual mean field scaling the Hamiltonian is further rescaled by $1 / N[6,3]$, in which case the error above reduces to $\mathrm{O}(q / 2 L+1)$.

We now consider a product binomial distribution as the prior distribution on the configuration space $\mathscr{H}_{m, q}$, i.e.

$$
P_{m, q}(\mathrm{~d} \eta)=\prod_{k=1}^{m} \rho_{q}(\mathrm{~d} \eta(k))
$$

where

$$
\rho_{q}(\eta(k)=\lambda)=\frac{q !}{\lambda !(q-\lambda) !}\left(\frac{1}{2}\right)^{q} .
$$

This prior arises naturally from the microscopic prior distribution in (2.3) by including $q$ independent sites. We define the canonical Gibbs measure on $\mathscr{H}_{m, q}$, as

$$
\mu_{m, q, \beta}(\mathrm{d} \eta)=\frac{1}{Z_{m, q, \beta}} \exp (-\beta \bar{H}(\eta)) P_{m, q}(\mathrm{~d} \eta)
$$


where $Z_{m, q, \beta}$ denotes the corresponding partition function. We readily see that if $q=1$, the measure reduces to (2.2). For brevity we use the following notation: for a fixed $\eta_{0} \in \mathscr{H}_{m, q}$, we write

$$
\begin{aligned}
& \mu_{m, q, \beta}\left(\left\{\eta: \eta=\eta_{0}\right\}\right):=\mu_{m, q, \beta}\left(\eta_{0}\right), \\
& P_{m, q}\left(\left\{\eta: \eta=\eta_{0}\right\}\right):=P_{m, q}\left(\eta_{0}\right), \\
& \rho_{q}\left(\eta(k)=\eta_{0}(k)\right):=\rho_{q}\left(\eta_{0}(k)\right) .
\end{aligned}
$$

Next we show that the coarse-grained process satisfies the detailed balance condition with respect to the measure $\mu_{m, q, \beta}$. The detailed balance for the process (3.19) would read

$$
c_{\mathrm{a}}(k, \eta) \mu_{m, q, \beta}(\eta)=c_{\mathrm{d}}\left(k, \eta+\delta_{k}\right) \mu_{m, q, \beta}\left(\eta+\delta_{k}\right)
$$

and

$$
c_{\mathrm{d}}(k, \eta) \mu_{m, q, \beta}(\eta)=c_{\mathrm{a}}\left(k, \eta-\delta_{k}\right) \mu_{m, q, \beta}\left(\eta-\delta_{k}\right) .
$$

First note that

$$
\bar{H}\left(\eta+\delta_{k}\right)-\bar{H}(\eta)=-\bar{U}(k)
$$

and

$$
\bar{H}\left(\eta-\delta_{k}\right)-\bar{H}(\eta)=\bar{U}(k)
$$

For simplicity in the exposition below we assume that $h=0$ and verify (4.5) only. Using (4.7) and the definitions of the rates (3.17), (3.18) we have

$$
\begin{aligned}
c_{\mathrm{a}}(k, \eta) \mu_{m, q, \beta}(\eta)-c_{\mathrm{d}}\left(k, \eta+\delta_{k}\right) \mu_{m, q, \beta}\left(\eta+\delta_{k}\right)= & (q-\eta(k)) \exp (-\beta \bar{H}(\eta)) P_{m, q}(\eta)-(\eta(k)+1) \\
& \times \exp \left(-\beta\left(\bar{H}\left(\eta+\delta_{k}\right)+\bar{U}(k)\right)\right) P_{m, q}\left(\eta+\delta_{k}\right) \\
= & \exp (-\beta \bar{H}(\eta))\left\{(q-\eta(k)) P_{m, q}(\eta)-(\eta(k)\right. \\
& \left.+1) P_{m, q}\left(\eta+\delta_{k}\right)\right\} \\
= & \exp (-\beta \bar{H}(\eta)) \prod_{l=1 \neq \neq}^{m} \rho_{q}(\eta(l))\left\{(q-\eta(k)) \rho_{q}(\eta(k))\right. \\
& \left.-(\eta(k)+1) \rho_{q}(\eta(k)+1)\right\} .
\end{aligned}
$$

However, from (4.3) we have that $(q-\lambda) \rho_{q}(\lambda)=(\lambda+1) \rho_{q}(\lambda+1)$ for all integers $0 \leqslant \lambda \leqslant q-1$. Thus the last curly bracket in (4.9) is identically zero and detailed balance follows.

\subsection{Large scale asymptotics of the invariant measure}

We derive an asymptotic formula for the invariant measure (4.4) as $q \rightarrow \infty$, in the spirit of calculations typically carried out as intermediate steps in the theory of Large Deviations [5].

First we recall Sterling's formula:

$$
q !=\sqrt{2 \pi q} \mathrm{e}^{-q} q^{q}(1+R(q)),
$$


where $1+R(q)=\exp \left(\theta_{q} / 12 q\right)$ and $0<\theta_{q}<1$. A standard calculation using (4.10) in (4.3) in the evaluation of all factorials yields (see for instance page 57 in [22]):

$$
\rho_{q}(\eta(k)=\lambda)=\frac{1+\epsilon(q, \lambda, q-\lambda)}{\sqrt{2 \pi q c_{k}\left(1-c_{k}\right)}} \exp \left(-q r\left(c_{k}\right)\right)
$$

or

$$
\rho_{q}(\eta(k)=\lambda)=(1+\epsilon(q, \lambda, q-\lambda)) \exp \left(-q\left(r\left(c_{k}\right)+\mathrm{o}_{q}(1)\right)\right),
$$

where

$$
1+\epsilon(q, \lambda, q-\lambda)=\frac{1+R(q)}{(1+R(\lambda))(1+R(q-\lambda))} .
$$

We have also defined the average coverage at $k \in \mathscr{L}_{\mathrm{c}}$,

$$
c_{k}=\frac{\lambda}{q}
$$

and

$$
r(c)=c \log c+(1-c) \log (1-c) .
$$

Using (4.11) we obtain the asymptotic formula for the measure (4.4). If $\eta_{0}=\left(\lambda_{1}, \lambda_{2}, \ldots, \lambda_{m}\right)$,

$$
\mu_{m, q, \beta}\left(\eta_{0}\right)=\prod_{k=1}^{m} \frac{1+\epsilon\left(q, \lambda_{k}, q-\lambda_{k}\right)}{\sqrt{2 \pi q c_{k}\left(1-c_{k}\right)}} \frac{1}{Z_{m, q, \beta}} \exp \left(-q m E_{m, q}\left(\eta_{0}\right)\right)
$$

which can be written as

$$
\mu_{m, q, \beta}\left(\eta_{0}\right)=\frac{1}{Z_{m, q, \beta}} \exp \left(-q m\left(E_{m, q}\left(\eta_{0}\right)+d\left(q, m, \eta_{0}\right)\right)\right)
$$

where

$$
E_{m, q}\left(\eta_{0}\right)=-\frac{\beta}{2 m \bar{L}} \sum_{l}\left\{\sum_{k \neq l} \bar{V}(k, l) c_{k} c_{l}+\bar{V}(0,0) c_{l}\left(c_{l}-\frac{1}{q}\right)\right\}+\frac{\beta h}{m} \sum_{k \in \mathscr{L}_{\mathrm{c}}} c_{k}+\frac{1}{m} \sum_{k \in \mathscr{L}_{\mathrm{c}}} r\left(c_{k}\right)
$$

or alternatively,

$$
E_{m, q}\left(\eta_{0}\right)=-\frac{\beta}{2 m \bar{L}} \sum_{l} \sum_{k} \bar{V}(k, l) c_{k} c_{l}+\frac{\beta h}{m} \sum_{k \in \mathscr{L}_{\mathrm{c}}} c_{k}+\frac{1}{m} \sum_{k \in \mathscr{L}_{\mathrm{c}}} r\left(c_{k}\right)+\mathrm{o}_{q}(1) .
$$

Here $\bar{L}=2 L+1 / q$ is the coarse-grained potential length of $\bar{V}$. Furthermore,

$$
d\left(q, m, \eta_{0}\right)=\frac{1}{q m} \sum_{k}\left\{\log \left(2 \pi q c_{k}\left(1-c_{k}\right)\right)+\frac{\theta_{q-\lambda_{k}}}{12 q\left(1-c_{k}\right)}+\frac{\theta_{\lambda_{k}}}{12 q c_{k}}\right\}-\frac{\theta_{q}}{12 q^{2}} .
$$

We notice that for fixed $m, d\left(q, m, \eta_{0}\right)=\mathrm{o}_{q}(1)$ as $q \rightarrow \infty$. Also observe that after some algebra (4.13) yields

$$
E_{m, q}\left(\eta_{0}\right)=\frac{\beta}{4 m \bar{L}} \sum_{l} \sum_{k} \bar{V}(k, l)\left[c_{k}-c_{l}\right]^{2}+\frac{\beta h}{m} \sum_{k \in \mathscr{L}_{\mathrm{c}}} c_{k}+\frac{1}{m} \sum_{k \in \mathscr{L}_{\mathrm{c}}}\left[r\left(c_{k}\right)-\beta \frac{V_{0}}{2} c_{k}^{2}\right]+\mathrm{o}_{q}(1),
$$


where

$$
V_{0}=\frac{1}{\bar{L}} \sum_{l} \bar{V}(k, l)
$$

Furthermore if $\bar{V}$ is long-ranged (i.e. $\bar{L}=m, N=2 L+1)$, (4.13) is merely a discrete version of the Lyapunov functional of the corresponding mesoscopic equation (4.17) (see Section 4.3 and [13]),

$$
E[c]=-\frac{\beta}{2} \iint V\left(y-y^{\prime}\right) c(y) c\left(y^{\prime}\right) \mathrm{d} y \mathrm{~d} y^{\prime}+\beta h \int c(y) \mathrm{d} y+\int r(c(y)) \mathrm{d} y .
$$

On the other hand if $\bar{V}=\bar{V}(k, l)$ is a nearest neighbor potential (i.e., $\bar{L}=1$ ) then (4.14) yields a discrete version of the well-known Ginzburg-Landau energy

$$
E[c]=\frac{\beta}{4 m^{2}} \int V_{0}|\nabla c|^{2}+\beta h \int c(y) \mathrm{d} y+\int r(c(y))-\frac{\beta}{2} V_{0} c^{2}(y) \mathrm{d} y .
$$

\section{Remarks}

1. The implication of (4.12) is that for large $q$ and $m$ fixed, the most probable configurations are the minimizers of the discrete energy (4.13). We recall that the minimizers of the continuous version (4.15) are standing waves, which for a specific choice of $J$ are known explicitely [8].

2. Using Laplace's principle [5] we may easily obtain Large Deviations results as $q \rightarrow \infty$ and as $q, m \rightarrow \infty$. In the former limit the rate is given in terms of (4.13) and in the latter in terms of (4.15). In all these derivations we need to guarantee that the "error" term $d$ in (4.12) vanishes as we pass to the limit. The full WKB expansion of the Gibbs measure can also be obtained easily from (4.12), in view of the explicit formula for $d$. Although we do not state explicitly either here or in Section 4.3 any theorems, it is straightforward to adapt our arguments, following the formalism of Large Deviations, into mathematically rigorous proofs.

\subsection{Comparison of microscopic and coarse-grained processes}

First we point out that the coarse-grained and the microscopic processes have the same deterministic mesoscopic limits. Indeed, for $g(\eta)=q^{-1} \eta(k)=\bar{\eta}(k)$ in (3.19) we can obtain using martingale arguments that in the $q, m \rightarrow \infty$ limit the same mesoscopic equation for Arrhenius dynamics as in the standard derivations from the microscopic process $\sigma,[13]$ :

$$
c_{t}=d_{0}[1-c-\exp (-\beta h) c \exp (-\beta J * c)]
$$

where $c$ is the limit of the normalized average $E \bar{\eta}_{t}(k)$. Thus the processes $\sigma$ and $\eta$ share the same deterministic mesoscopic limit. Details of the asymptotic limit will appear elsewhere.

In addition, we would like to make a comparison of the probability distribution functions (PDF) for the coarse-grained and microscopic processes. Here we discuss only the case of the corresponding invariant measures (2.2) and (4.4) and return to the issue for the full non-equilibrium setting in a future publication.

First, it is immediately obvious that the Gibbs measures (2.2) and (4.4) are identical when the coarsegraining parameter $q$ is equal to one. Next we examine their relation in the asymptotic limits $m \rightarrow \infty$, $q \rightarrow \infty$. Recall that in Section 3 we defined the mapping $F: \Sigma \mapsto \mathscr{H}_{m, q}$, where

$$
F(\sigma)(k)=\sum_{y \in D_{k}} \sigma(y)=\eta(k), \quad k=1, \ldots, m .
$$


Following along the lines of the calculations in Section 4.2 and using the simple probabilistic fact that $P_{N}\left(\left\{\sigma: F(\sigma)=\eta_{0}\right\}\right)=P_{m, q}\left(\eta_{0}\right)$, we have:

$$
\begin{aligned}
\mu_{\mathscr{L}, \beta}\left(\left\{\sigma: F(\sigma)=\eta_{0}\right\}\right) & =\frac{1}{Z_{\mathscr{L}, \beta}} \int_{\left\{\sigma: F(\sigma)=\eta_{0}\right\}} \exp (-\beta H(\sigma)) P_{N}(\mathrm{~d} \sigma) \\
& =\frac{1}{Z_{\mathscr{L}, \beta}} \exp \left(-\left(\beta \bar{H}\left(\eta_{0}\right)+\mathrm{O}(N q / 2 L+1)\right)\right) P_{N}\left(\left\{\sigma: F(\sigma)=\eta_{0}\right\}\right) \\
& =\frac{1}{Z_{\mathscr{L}, \beta}} \exp \left(-m q\left(E_{m, q}\left(\eta_{0}\right)+d\left(q, m, \eta_{0}\right)+\mathrm{O}(q / 2 L+1)\right)\right) \\
& =\frac{1}{Z_{\mathscr{L}, \beta}} \exp \left(-m q\left(E_{m, q}\left(\eta_{0}\right)+d\left(q, m, \eta_{0}\right)+\mathrm{O}(1 / \bar{L})\right)\right)
\end{aligned}
$$

Consequently, for fixed $m$ and $\bar{L}$, and $q \rightarrow \infty$, the discrete energy (4.13) corrected by the term $\mathrm{O}(1 / \bar{L})$ is the rate functional of the underlying Large Deviation principle. In the consecutive $q, m \rightarrow \infty$ (hence $\bar{L} \rightarrow \infty$ ) the rate functionals of $\mu_{m, q, \beta}$ and $\mu_{\mathscr{L}, \beta}$ are identical, yielding asymptotically identical PDFs for the stationary measures of the microscopic and coarse-grained processes. In fact, in the case of long range interactions $(N=2 L+1)$ we have that $(4.15)$ is the rate functional of both processes. We note here that this last result was first proved for the microscopic Gibbs states in [6]. Contrasting relations (4.12) and (4.18) allow us, at least at equilibrium, to rationally design the coarse-grained Monte Carlo simulation, i.e., decide how to select $m$ and $q$, given a potential $J$ and a desired accuracy. This comparison of fluctuations in microscopic and coarse-grained processes is also numerically demonstrated in a non-equilibrium setting with an illustrative example in Section 5.

\section{Coarse-grained Monte Carlo simulations}

\subsection{Implementation of kinetic MC simulations}

Given a microscopic potential, the coarse-grained potential between interacting coarse cells is first computed only once and saved in a vector. This makes the computational demand of coarse-grained MC simulations per event the same as that of microscopic MC simulations. More specifically we implement (3.8) in the most straightforward manner as

$$
\bar{J}(k, l)=\frac{1}{q^{2}} \sum_{x \in D_{k}} \sum_{y \in D_{l}} J(x-y)=\frac{1}{2 L+1} \bar{V}(k, l), \quad k \neq l,
$$

where we define

$$
\bar{V}(k, l)=\frac{1}{q^{2}} \sum_{x \in D_{k}} \sum_{y \in D_{l}} V\left(\frac{N|x-y|}{2 L+1}\right), \quad k \neq l .
$$

Here we have a total of $q^{2}(x, y)$-pairs corresponding to $q^{2}$ pairwise interactions. Likewise, within the same cell we have $q(q-1) / 2$ distinct pairs of interactions; for all $l=1,2, \ldots, m$,

$$
\bar{J}(l, l)=\bar{J}(0,0)=\frac{1}{q(q-1)} \sum_{x \in D_{l}} \sum_{\substack{y \in D_{l} \\ y \neq x}} J(x-y) .
$$


Similarly we set

$$
\bar{V}(l, l)=\bar{V}(0,0)=\frac{1}{q(q-1)} \sum_{x \in D_{l}} \sum_{\substack{y \in D_{l} \\ y \neq x}} V\left(\frac{N|x-y|}{2 L+1}\right) .
$$

With the exception for the aforementioned interactions within a coarse cell, which are not a feature of microscopic MC simulations, coarse-grained MC simulations are implemented in the same manner. The primary differences lie in the occupation function $\eta(k)$ that varies from 0 to $q$ instead of having two values of 0 and 1, and in computing the Hamiltonian where we have to include the term describing the interactions within the cell. As a result, the transition probabilities and average time step (the inverse of the total transition probability per unit time) are also scaled appropriately. The updating mechanism either adds or subtracts a particle at each coarse cell in the case of adsorption/desorption, or moves a particle between adjacent coarse cells in the case of surface diffusion (see Appendix A). However, for both mechanisms the maximum number of particles at each coarse cell is $q$ and the minimum is zero. For instance, this exclusion principle is enforced in (A.13) by the prefactor $\eta(k)(q-\eta(l))$. The computational implication of this is that physically unrealistic situations (e.g., fraction of occupied sites greater than one or less than zero) never arise.

We recall from Section 2.1 that $L$ denotes the potential radius of the microscopic potential $J$ (in microscopic units). Then $\bar{L}=2 L+1 / q$ is the total range of interaction of the coarse-grained potential $\bar{J}$. The system modeled is a $1 \mathrm{D}$ surface (interface) in equilibrium with a fluid phase, as depicted in the inset of Fig. 1. Atoms adsorb from the fluid onto the interface and can desorb back, depending on temperature and specifics of interactions between atoms. We denote the adsorption and desorption constants with $k_{\mathrm{a}}$ and $k_{\mathrm{d}}$, respectively, as well as $P$ the constant partial pressure in the gas phase. These parameters enter in the MC rates (2.6) as follows: $k_{\mathrm{d}}=d_{0}, k_{\mathrm{a}} P=\exp (-\beta h)$, where $h$ is the external field in (2.5). The expected value of $\eta(k)$ normalized by $q$ is called the coverage $u$. For equilibrium systems with periodic boundary conditions, the coverage reported below is the ensemble (spatial) average.

The algorithm we have implemented belongs to the general class of continuous time MC (CTMC) or often referred to as kinetic MC simulations. In these algorithms, the transition probabilities are computed a priori and using one or more random numbers, a microscopic process is selected and executed. As a result, there are no null events in which no action takes place. Since the probability of having successful events is one, these algorithms can be superior to null event algorithms. Several of these algorithms along with their performance have recently been discussed in [21]. Here we employ a global update scheme, i.e., at each MC event a site is randomly chosen and the lattice is scanned until the selected site is identified. In a typical MC simulation, the following steps are carried out: (1) calculation of the transition probabilities per unit time of each site for the entire lattice; (2) selection of a microscopic process, either adsorption or desorption, and subsequently of a specific site using a random number and the transition probabilities and (3) execution of the event at the selected site. Since the time step is inversely proportional to the number of cells, in order to conduct simulations of the same real time or MC steps (MCS), the lattice is scanned once on the average (we define this as 1 MCS).

Finally we remark that a local update algorithm could also be implemented. In local update algorithms, sites with the same transition probability are assigned to the same class or set of sites. Furthermore, only the sites whose probabilities are affected after the execution of an event are updated, i.e., within a radius of $L$. By using lists of neighbors and saving their coordinates, one can directly identify the selected site and eliminate scanning of the lattice, resulting in significant computational savings in many cases [21]. Implementation of local update algorithms is however tedious and memory can become a serious issue, especially for long-range potentials, as the number of needed classes increases rapidly. For these reasons, we have employed the simpler global update algorithm. Note though that the coarse-grained MC methods proposed 
here can effectively shorten the microscopic potential length, enabling in some cases the use of local update algorithms even for long-range potentials.

For most of the simulations reported below, a microscopic piecewise constant potential is employed to describe lateral adsorbate-adsorbate interactions. It is defined in terms of (2.4) for $V(x)=V(r), x \in \mathbf{R}$, $|x|=r$ and

$$
V(r)=J_{0} \chi_{[0,1]}(r), \quad r \geqslant 0,
$$

where $\chi_{[0,1]}(r)=1$, if $0 \leqslant r \leqslant 1$, and $\chi_{[0,1]}(r)=0$, otherwise. The convention used below is that $J_{0}$ is positive or negative, for attractive or repulsive interactions, respectively. The coarse-grained potential $\bar{J}$ is defined as in (5.1).

\subsection{Isotherms}

It is known from statistical mechanics that no hysteresis exists for first-nearest neighbor attractive interactions in 1D systems [12]. On the other hand, for infinitely long attractive interactions, hysteresis exists according to the global mean field (MF) theory for $\beta J_{0}>4$, where $J_{0}$ is the zeroth moment of the potential, [10]. Therefore, it is important to understand at what potential lengths hysteresis develops in 1D simulations. Furthermore, we would like to investigate whether very short-range coarse-grained MC simulations give hysteresis for relatively long microscopic potentials. This constitutes a strict test that coarse-graining works well for metastability and hysteresis.

Isotherms are computed similarly to natural parameter continuation, i.e., we trace the coverage vs. pressure (called an isotherm), first upon increasing $k_{\mathrm{a}} P / k_{\mathrm{d}}$ from low values and then on decreasing it from high values. For each calculation at a new pressure the final state from the previous pressure is used as an initial configuration. Fig. 1 compares the global MF isotherm with those obtained from microscopic MC simulations for sufficiently strong interactions $\left(\beta J_{0}=6\right)$ and various potential lengths $L$. For the microscopic MC simulations, no hysteresis exists even for $L=10$. Hysteresis is observed though for sufficiently long potentials such as $L=20$ and $L=30$. The hysteresis width increases with increasing potential length towards the global MF limit, as expected.

Next, gross features of microscopic $(q=1)$ and coarse-grained $(q>1)$ MC simulation data are compared in Fig. 2 for a fixed potential length and various coarse-graining levels. First we notice that the coverage predicted from the coarse-grained MC simulation is in reasonably good agreement with that of the microscopic MC simulation along each branch of the isotherm. However, small quantitative differences are observed. We discuss these quantitative errors and their evolution with increasing $q$ in detail below. Second we remark that, as the asymptotics (3.13) indicate, for fairly long potentials (e.g., $L=30$ ) the coarse-grained MC predicts hysteresis in good agreement with the microscopic MC simulation. This is even true when the asymptotics (3.13) are not necessarily true, for instance in first nearest neighbor coarsegrained interactions $(q=2 L+1)$. These simulations demonstrate that despite the fact that the coarsegrained MC procedure results in short-range interactions, it correctly predicts hysteresis in $1 \mathrm{D}$ for relatively long potentials. Therefore, the coarse-grained MC simulation provides the correct qualitative behavior.

The small differences in pressure where the transition from low to high coverages occurs upon increasing $k_{\mathrm{a}} P / k_{\mathrm{d}}$ are due in part to the stochastic nature of these simulations associated with metastability in hysteresis loops and in part due to the approximation of the coarse-grained potential and energies compared to the microscopic MC simulation. It is also noticed that the larger $q$ is, the more global MF-like behavior is observed. As the level of coarse-graining increases, more microscopic cells are averaged within a coarse cell, i.e., each cell behaves more like a MF entity and the interactions between coarse cells decrease in magnitude and probably in length. For sufficiently large coarse-grainings, one would expect that the global MF limit is recovered, except for fluctuations, as also discussed at the end of Section 3.1. Fig. 2 shows an example for 


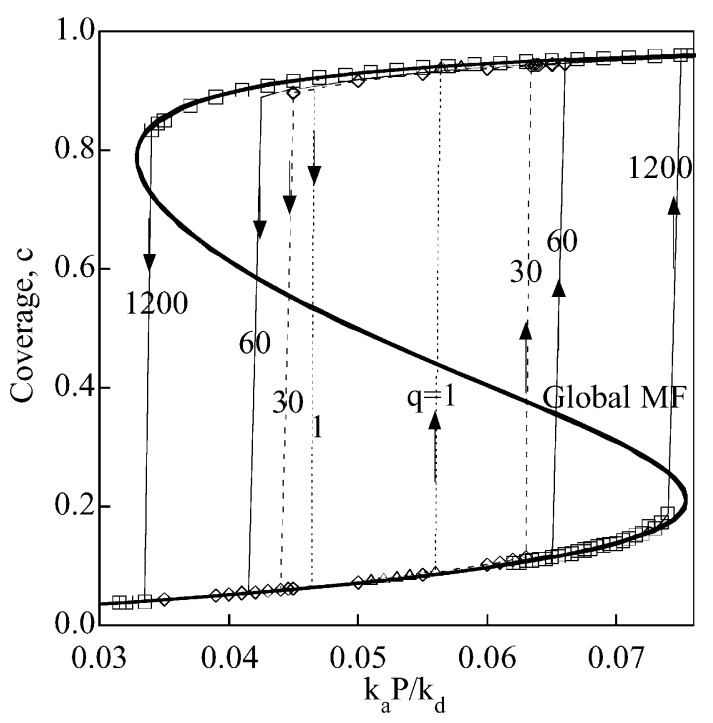

Fig. 2. Comparison of coarse-grained and microscopic MC simulation results for $L=30$ and different levels of coarse-graining $q$ for a total number of cells $N=m q=240$ (for $q>30, m=20$ has been used). The global MF isotherm is also depicted as a thick solid line. For the largest coarse-grainings, long simulations of up to $10^{6} \mathrm{MCS}$ have been carried out near turning points. Other parameters are the same as in Fig. 1.

$q=1200$ where interactions are limited to almost within each cell. Indeed the coarse MC isotherm (squares) almost completely coincides with the MF isotherm. However, slightly premature transitions from one branch to the other are still observed caused by the noise inherent to the stochastic process. Such transitions are observed only in very long MC simulations and take considerable number of MC steps $\left(>10^{6}\right)$ to transition from one branch to the other, a situation reminiscent of critical slow down of continuum PDEs. For example, shorter runs of $10^{4}$ do not exhibit such premature transitions.

The above simulations were done for moderately long potentials. Fig. 3 shows isotherms for very shortrange potentials $(L=1)$. In contrast to the multi-valued global MF isotherm, the microscopic MC isotherm (circles connected with line) is single-valued, as expected from statistical mechanics. For such short-ranged potentials, significant deviations are observed for coarse-grainings varying from $q=2$ to $q=24$, but the corresponding isotherms are still single-valued. For $q=24$ the isotherm is quite steep, and for larger coarse-grainings, such as $q=100$ and $q=1000$, the MF character within each coarse cell dominates and multiple-valued isotherms develop as indicated in Fig. 3. Note though that despite the small value of $\bar{L}=2 L+1 / q \ll 1$, fluctuations can result in premature transitions from one branch to the other near turning points, a feature which is absent from deterministic ODEs. The situation about the effect of fluctuations is analogous to one seen in Fig. 2. However, similarly to the mean field theory, coarse-graining may lead to qualitatively wrong results (in terms of multiplicity) for short potentials in 1D.

\subsection{Transients and noise of coarse-grained MC simulations}

The above simulations provide ensemble averages of equilibrium states. There are several interesting facets to explore in transients such as the correctness of dynamics towards equilibrium and the level of noise. Single MC transient runs were carried out with periodic boundary conditions for three cases: no interactions (Langmuir case), attractive interactions (highest coverage) and repulsive interactions (lowest coverage). In the case of interactions, a piecewise constant potential of $L=10$ has been used. Results are 


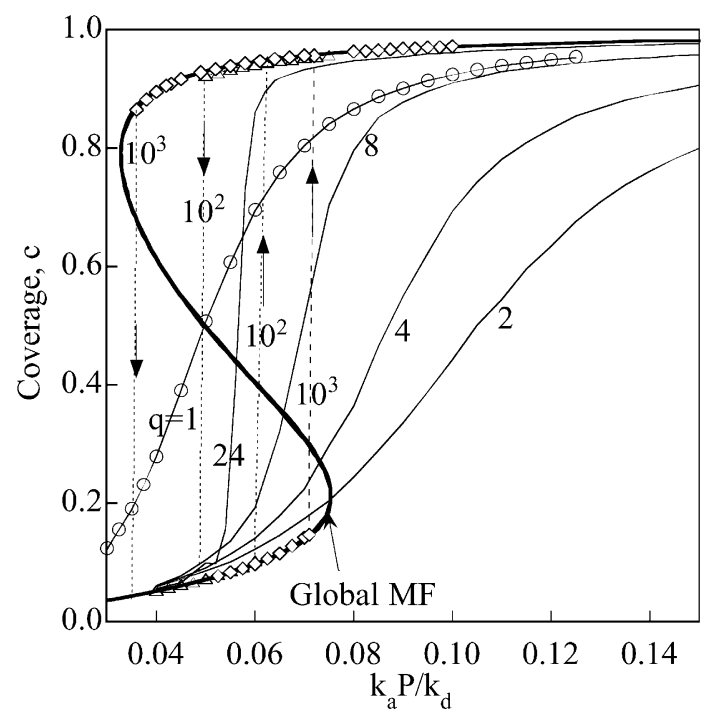

Fig. 3. Comparison of global MF, microscopic MC simulation, and coarse-grained MC simulation results for short-range potentials of $L=1$ and different levels of coarse-graining $q$ for a total number of cells $N=m q=240$ (for $q>30, m=20$ has been used). The rest of the parameters are the same as in Fig. 1. For the largest coarse-grainings, long simulations of up to $10^{6}$ MCS have been carried out near turning points.

depicted in Fig. 4. The solid lines correspond to the microscopic MC and the dashed ones to coarse-grained MC with $m=200$ and $q=10$. The smooth dashed line is the Langmuir transient corresponding to the global MF theory in the absence of interactions (an analytic solution). The same seed of the random generator has been used for all runs displayed in Fig. 4.

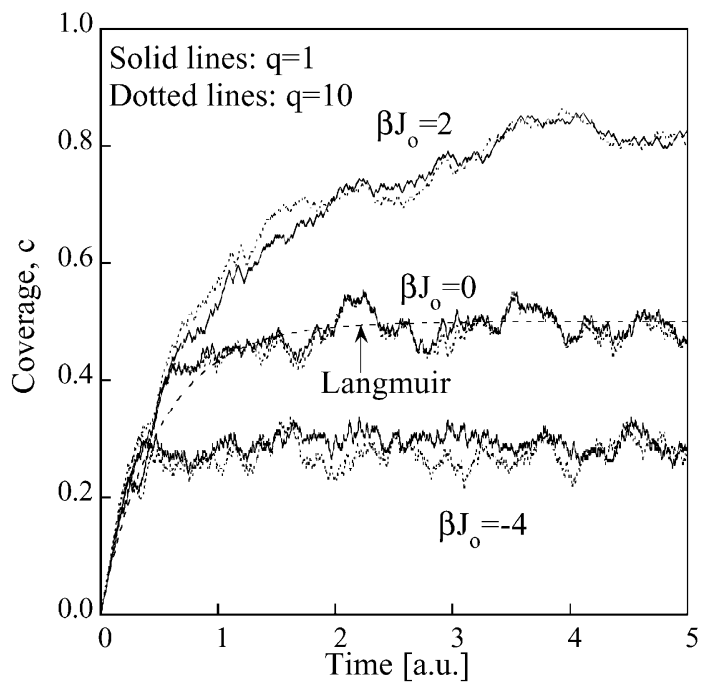

Fig. 4. Results of transient simulations from microscopic $(q=1) \mathrm{MC}$ (solid, noisy lines) and coarse-grained ( $q=10) \mathrm{MC}(\mathrm{dotted}$, noisy lines) for an attractive, a repulsive, and a non-interacting case. The smooth dashed line is the analytic Langmuir result for $J_{0}=0$. The other parameters are: $k_{\mathrm{a}} P=k_{\mathrm{d}}=1, m=200, L=10$. 
The model responses are noisy, as expected from single MC runs. The coarse-grained MC data follows closely the microscopic MC data, i.e., the correct dynamics is captured. What is more astonishing is that the noise of the two simulations is comparable (comparison of the noise is the reason we do not average multiple trajectories to smooth these curves). Thus, the coarse-grained MC describes correctly the system dynamics and exhibits the same noise as the microscopic MC simulation. This fact is not surprising, for instance the Gibbs states of the coarse-grained process and the underlying microscopic process are asymptotically identical in the case of long range interactions $(2 L+1=N)$, as the Large Deviation principles for the coarse-grained and the microscopic processes coincide asymptotically (see (4.12) and (4.18)).

\subsection{Errors caused by coarse-graining and effect of detailed balance}

In order to examine the accuracy of the hierarchy of the coarse-grained MC simulations, beyond the rigorous asymptotics presented in Sections 3 and 4, we have performed a number of simulations for various values of the strength and length of the potential vs. cell size $q$. The results for piecewise constant potential are depicted in Fig. 5. The top graph corresponds to repulsive interactions and the bottom one to attractive interactions. In all simulations $L$ is kept fixed (we study two cases, $L=1$ and $L=16$ as representative of very short interactions and medium range interactions) while $q$ gradually increases from the microscopic $q=1$ limit to global mean field limit $q \gg L$.

Several interesting features are noticed. As $q$ increases from the microscopic MC value of $q=1$ to large values, the coverage does not always change monotonically (see $L=1$ case in Fig. 5(a) where the coverage starts from 0.2855 at $q=1$ ) but it may exhibit maximum deviations from the microscopic MC value for intermediate coarse grainings. For large $q$, mean field behavior is achieved within each cell and the global

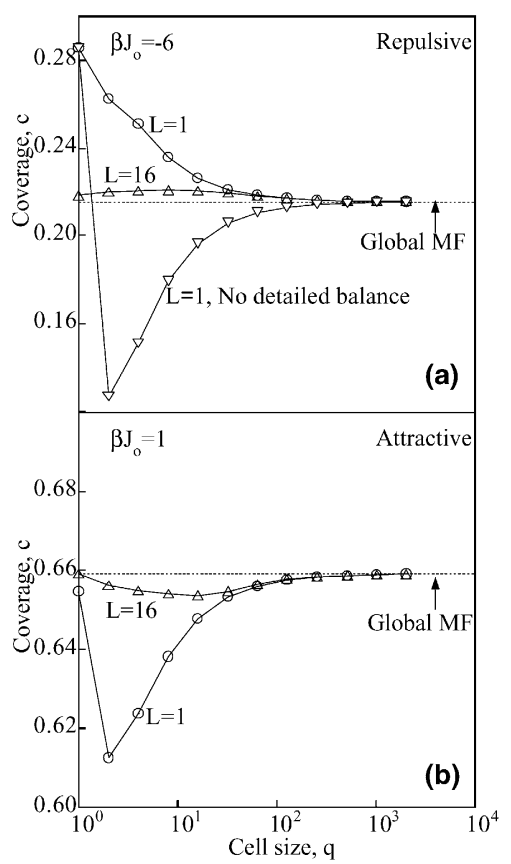

Fig. 5. Errors of coarse-grained MC simulations vs. cell size $q$ for repulsive interactions (a) and attractive interactions (b). The parameters used are: $k_{\mathrm{a}} P=k_{\mathrm{d}}=1$ with ensemble averaging of $10^{4} \mathrm{MCS}$ after equilibration. Here $m=256$ is fixed as $q$ increases (variable $N)$. An example where no detailed balance is satisfied is also shown for repulsive interactions in panel (a). 
mean field value (0.2154 in Fig. 5(a)) is recovered, as expected. For short-range potentials, deviations of up to $20 \%$ are noticed. These deviations are worst for short-range potentials and in most cases are nonmonotonic. Furthermore, the value of $q$ where the maximum deviations are observed (for the nonmonotonic cases) changes with potential length.

The detailed balance principle has been used as a design rule of coarse-graining processes instead of other intuitive approaches. In order to delineate the importance of this issue, we have also carried out simulations where the short range interaction term in $(4.1) \eta(l)(\eta(l)-1)$ has been deliberately replaced with another "intuitive" term $\eta(l)^{2}$, which however does not satisfy detailed balance. An example of the effect of lack of detailed balance is depicted in Fig. 5(a). In this case large discrepancies are observed from the results where detailed balance was enforced. Therefore, the correct derivation of the coarse Hamiltonian from the microscopic one can be critical regarding numerical accuracy. This error is especially important for relatively small $q$; in contrast, the two interaction terms tend to each other for large coarse grainings and large coverages. Fig. 6 elucidates this point by comparing isotherms for a very short-range potential $L=1$. For $q=2$ and low coverages, the deviations are significant. For larger $q$ and larger coverages, the deviations are less important.

\subsection{Morse potentials}

In addition to the piecewise constant potential used above, we have carried out a number of simulations using the Morse potential, which is given by

$$
V(x)=-A\left[-2 \exp \left(\frac{x_{0}-x}{b}\right)+\exp \left(\frac{2\left(x_{0}-x\right)}{b}\right)\right], \quad x \geqslant 0 .
$$

The potential is depicted in Fig. 7. Here $A$ is the energy at $x=x_{0}, x_{0}$ determines the distance to the minimum of the potential (equilibrium point) and $b$ is the curvature at the equilibrium point that mainly controls the

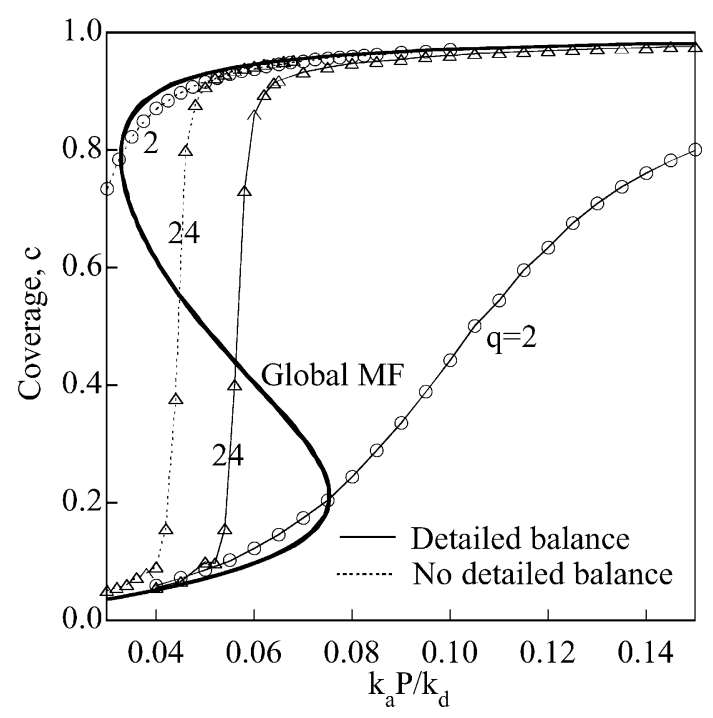

Fig. 6. Comparison of coarse-grained MC isotherms with and without detailed balance for short-range potentials of $L=1$ and different levels of coarse-grainings $q$ for a total number of cells $N=m q=240$. The global MF isotherm is also shown. The rest of the parameters are the same as in Fig. 3. 


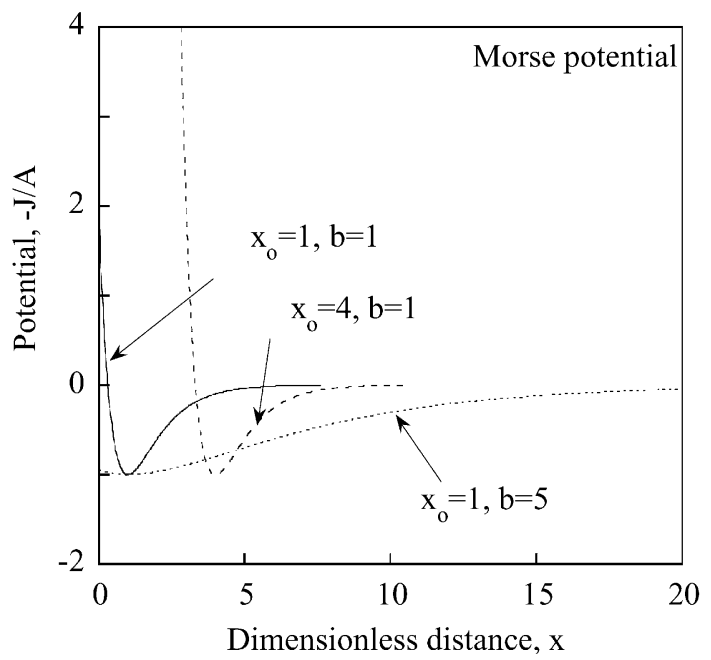

Fig. 7. Morse potential as a function of distance for different parameter values indicated for each curve.

rate of decay of the potential with distance toward zero, i.e., the range of interactions. The effective range (cutoff distance) of the Morse potential varies with parameters but it is chosen to be in the range of 20-40 neighbors. Note that for positive $A$, the interactions are attractive, whereas for negative $A$ the interactions are repulsive.

Fig. 7 depicts the potential vs. distance for three choices of parameters. Two choices correspond to potentials where the minimum is at $x_{0}=1$ but the potential range differs by adjusting $b$. The last choice corresponds to $x_{0}=4$ so that shorter-range interactions are repulsive and longer-range interactions are attractive. The errors using these sets of parameters as a function of the cell size $q$ are depicted in Fig. 8. The top and bottom graphs have been obtained for opposite values of the potential strength $A$. The value of $A$ has been adjusted in each of the three sets of parameters in order to maintain the absolute value of the zeroth moment of the potential constant in all these simulations.

We notice that the relative errors are relatively small, i.e., the coarse graining idea works well even for non-piecewise constant potentials. The overall behavior regarding the error is similar to the piecewise constant potential, i.e., the maximum error occurs for intermediate values of $q$, and global MF behavior is recovered for large $q$. Furthermore, the error is smaller for longer range potentials (see the case of $b=5, x_{0}=1$, triangles). Finally, the error is bigger for mixed type potentials (see the case of $b=1, x_{0}=4$, squares) where attractive and repulsive interactions are averaged within each cell.

\section{6. $C P U$ issues}

Table 1 summarizes the number of function evaluations needed in each MC event. In this algorithm, almost the entire CPU is spent on computing the transition probabilities. Among these calculations, the

Table 1

Operation counting

\begin{tabular}{lll}
\hline Step & Number of multiplications and divisions & Number of exponentiations \\
\hline Calculation of transition probabilities & $m+2 m+2 L /(q m)=m(2 L / q+3)$ & $m$ \\
Site selection & $\mathrm{O}(m)$ to $\mathrm{O}\left(\operatorname{lnm}^{1 / 2}\right)$ & \\
Execution of event & 1 & \\
\hline
\end{tabular}

We account for 'if statements' as multiplications. Details depend on specifics of implementation. 


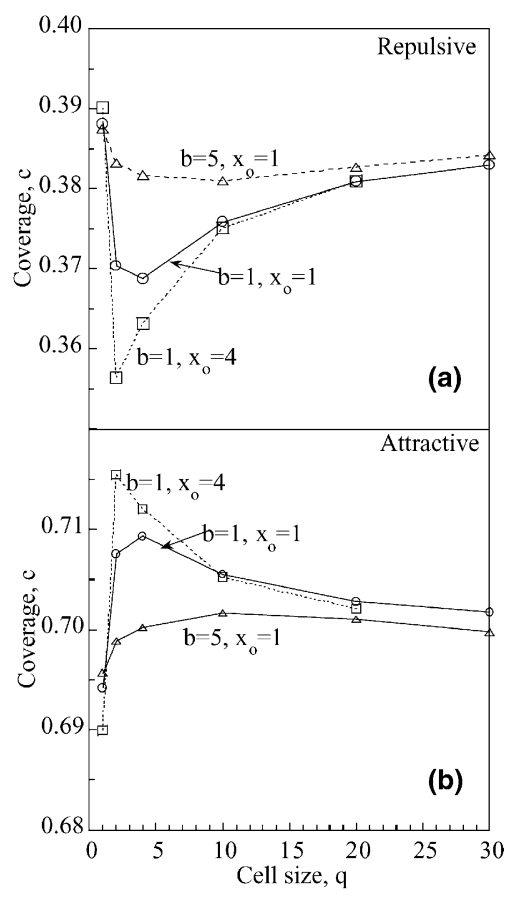

Fig. 8. Coverage vs. cell size $q$ for net repulsive interactions (top) and attractive interactions (bottom) using the Morse potential and three sets of parameters used in Fig. 8. Points are simulation results and lines just connect the points. The behavior is similar to the piecewise constant potential. The largest errors occur for short-range, mixed type of potentials (squares) and the smallest ones for the longer-range potentials (triangles). In these simulations, the strength, $A$, of the Morse potential at the minimum has been adjusted so that the absolute value of the zeroth moment of the potential remains constant in all simulations.

exponentiation and the computation of the convolution (energy term) are the most demanding operations, depending on the potential length. The selection process has been reported to vary from $\mathrm{O}\left(\ln \left(m^{1 / 2}\right)\right)$ to $\mathrm{O}(m)$ when rare events are encountered. Given the function evaluations, the CPU per MC step (keeping the same real time as the number of cells varies) is expected to vary as $m^{2}$ and be proportional to $L$.

For a given simulation length $N$ and a fixed potential length $L$, as $q$ increases, the number of coarse cells $m=N / q$ decreases. Furthermore, the scale of the coarse-grained potential decreases as $q / 2 L+1$. Therefore, for sufficiently long potentials, the CPU time decreases with increasing $q$ approximately as $\mathrm{O}\left(1 / q^{3}\right)$ or $\mathrm{O}\left(m^{3}\right)$. Fig. 9 shows this dependence. The CPU scales with $m^{2.87}$. As an example, using $q=100$, it results in approximately 1 million speedup of the code. Therefore, for relatively long range potentials, significant enhancement in performance is obtained. For macroscopic size systems in the millimeter length scale or larger, microscopic MC simulations are impractical on a single processor. The computational savings of coarse-grained MC make it a suitable tool for such scales.

Finally, note that the execution CPU time is the same for the microscopic and coarse-grained MC for each event, as far as the same number of simulated cells $m$ and the same potential length are employed. The time step in KMC is inversely proportional to the number of simulated cells [21]. For the same simulated length, $N=m q$ (in microscopic lattice units), as $q$ increases the number of cells $m$ decreases. However, the transition probabilities per unit time increase proportionally with $q$. Therefore, the time step is independent of coarse graining. This is an expected result, as individual atoms only keep moving 


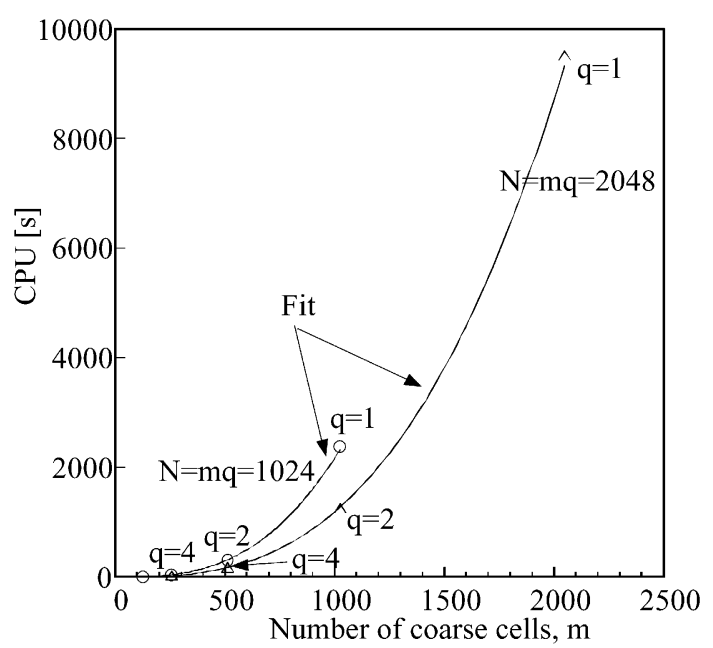

Fig. 9. CPU (in seconds) requirements vs. the number of coarse-grained cells, $m$, for two fixed total number of cells, $N=m q=1024$ and $N=2048$, and a fixed number of $100 \mathrm{MCS}$. The parameters used are $k_{\mathrm{a}} P=k_{\mathrm{d}}=1, \beta J_{0}=2$, and $L=256$. The points are actual data and the lines are power law fits. An almost cubic dependence of CPU on the number of cells is observed for large domains and long potentials.

at each $\mathrm{MC}$ event in both simulations. Therefore coarse-graining presented here applies to length rather than time.

\section{Conclusions}

In this paper we have introduced a new class of coarse-grained stochastic processes and associated Monte Carlo simulations which are derived directly from microscopic lattice systems and describe mesoscopic length scales. Detailed balance is employed as a systematic design principle to guarantee proper inclusion of noise fluctuations in the coarse-grained model. Asymptotic analysis using Large Deviations techniques and numerical comparisons of coarse-grained and conventional-microscopic-Monte Carlo (MC) simulations delineate the validity regimes of the proposed coarse-graining procedure. It is also demonstrated that the new models result in significant computational savings by reducing the cost of the microscopic MC simulations by a factor of approximately $q^{3}$, where $q$ is the size of the coarse-graining. Consequently the proposed coarse-grained MC simulations are capable of capturing large scale features, while retaining microscopic information on intermolecular forces and particle fluctuations.

The proposed algorithms have the potential for significant impact on numerous technologically relevant applications which are currently intractable with conventional MC simulations. Examples include pattern formation at mesoscopic length scales on catalytic surfaces [11,14], transport through microporous films [24], as well as growth processes of materials. Furthermore coarse-grained MC methods can provide a new tool for the simulation of systems having a wide discrepancy of interrelated scales. One such process is Chemical Vapor Deposition where micrsocopic interfacial phenomena typically simulated by MC methods affect the large scale adjacent fluid flow [23]. In the same broad multiscale context but in an entirely different direction, coarse-grained stochastic processes can be employed as mesoscopic stochastic models for unresolved features in atmospheric phenomena. For example such models and MC simulations can be directly derived from microscopic stochastic models for the parameterization of tropical convection developed recently in [19]. 
From both a mathematical and applied perspective it is important to investigate further other coarsegrainings which are designed for specific interaction potentials, based for instance on the the wavelet approach outlined in Section 3.3. In the rigorous analysis part of this paper we focused on error estimates and asymptotics between the invariant measures of the coarse-grained and the microscopic processes; a followup step in this direction is the comparison between the probability distribution functions of the two processes as well as an explicit Large Deviation analysis, in non-equilibrium.

\section{Acknowledgements}

The research of M.A.K. is partially supported by NSF-DMS-0079536, NSF-DMS-0100872, and NSF-ITR-0219211, the research of A.J.M. is partially supported by ONR N00014-96-1-0043, NSF-DMS9972865, and ARO-DAAD19-01-10810 and the research of D.G.V. is partially supported by NSF-CTS9904242 and NSF-ITR-0219211. M.A.K. and A.J.M. would like to thank the Institute for Pure and Applied Mathematics at the University of California, Los Angeles, where part of this work was carried out during visits in May 2001 and January 2002.

\section{Appendix A. Coarse-grained stochastic processes for surface diffusion}

A spin exchange between the neighboring sites $x$ and $y$ is a spontaneous exchange of the values of the order parameter at $x$ and $y$. Physically this mechanism describes the diffusion of a particle on a flat surface. Note that sites cannot be occupied by more than one particle (exclusion principle). As in the spin-flip dynamics, a spin exchange occurs with rate $c(x, y, \sigma)$ satisfying the detailed balance law

$$
c(x, y, \sigma)=c\left(x, y, \sigma^{(x, y)}\right) \exp \left(-\beta \Delta_{(x, y)} H(\sigma)\right),
$$

where $\sigma^{(x, y)}$ is the new configuration after a spin exchange between sites $x$ and $y$

$$
\sigma^{(x, y)}(z)= \begin{cases}\sigma(y) & \text { when } z=x \\ \sigma(x) & \text { when } z=y \\ \sigma(z) & \text { otherwise }\end{cases}
$$

Furthermore

$$
\Delta_{x, y} H(\sigma)=H\left(\sigma^{(x, y)}\right)-H(\sigma)=(\sigma(x)-\sigma(y))(U(x)-U(y))
$$

is the energy difference after performing a spin exchange between the neighboring sites $x$ and $y$, and

$$
U(x)=\sum_{z \neq x} J(x-z) \sigma(z)-h
$$

is the total energy contribution from the particle interactions with the particle located at the site $x$.

The resulting stochastic process $\left\{\sigma_{t}\right\}_{t \geqslant 0}$ is a jump Markov process on $L^{\infty}(\Sigma ; \mathbf{R})$ with generator given by

$$
M_{N} f(\sigma)=\sum_{x, y \in \mathscr{L}} c(x, y, \sigma)\left[f\left(\sigma^{(x, y)}\right)-f(\sigma)\right] .
$$

The simplest type of dynamics satisfying (A.1) is the Metropolis-type dynamics

$$
c(x, y, \sigma)= \begin{cases}\Psi\left(-\beta \Delta_{x, y} H(\sigma)\right), & \text { when } x \text { and } y \text { are nearest neighbors, } \\ 0 & \text { otherwise, }\end{cases}
$$


where $\Psi(r)=\Psi(-r) \mathrm{e}^{-r}, r \in \mathbf{R}$. Typical choices of $\Psi$ 's are $\Psi(r)=2\left(1+\mathrm{e}^{r}\right)^{-1}$ (Kawasaki dynamics) and $\Psi(r)=\mathrm{e}^{-r^{+}}$(Metropolis dynamics). As in the adsorption/desorption case for such dynamics, the energy barrier for diffusion depends only on the energy difference between the initial and final states, often known as the heat of the process.

On the other hand, in Arrhenius-type dynamics the activation energy of surface diffusion is defined as the energy barrier a species has to overcome in jumping from one site to another [24]. The Arrhenius surface diffusion (spin exchange) rate is given for nearest neighbors $x$ and $y$ by

$$
c(x, y, \sigma)= \begin{cases}c_{0} \exp \left[-\beta\left(U_{0}+U(x)\right)\right] & \text { when } \sigma(x)=1, \sigma(y)=0, \\ c_{0} \exp \left[-\beta\left(U_{0}+U(y)\right)\right] & \text { when } \sigma(x)=0, \sigma(y)=1, \\ 0 & \text { otherwise }\end{cases}
$$

or equivalently

$$
c(x, y, \sigma)=c_{0} \sigma(x)(1-\sigma(y)) \exp \left[-\beta\left(U_{0}+U(x)\right)\right]+c_{0} \sigma(y)(1-\sigma(x)) \exp \left[-\beta\left(U_{0}+U(y)\right)\right],
$$

where $U(x)$ is given by (A.3) while $U_{0}$ is the energy associated with the surface binding of the particle at $x$ and $c_{0}$ is a rate constant that can be chosen arbitrarily. Arrhenius dynamics also satisfy the detailed balance law.

Here we proceed similarly to the spin-flip dynamics and define the coarse-grained process on $\mathscr{L}_{\mathrm{c}}$,

$$
\eta_{t}(k)=\sum_{y \in D_{k}} \sigma_{t}(y), \quad k=1, \ldots, m,
$$

set on the configuration space

$$
\mathscr{H}_{m, q}=\{0,1, \ldots, q\}^{\mathscr{L}_{\mathrm{c}}},
$$

where $\eta=\left\{\eta(k): k \in \mathscr{L}_{\mathrm{c}}\right\}$ and $\eta(k) \in\{0,1, \ldots, q\}$ is the coverage of the coarse cell $D_{k}$. We also define the mapping $F: \Sigma \mapsto \mathscr{H}_{m, q}$, where

$$
F(\sigma)(k):=\sum_{y \in D_{k}} \sigma(y)=\eta(k), \quad k=1, \ldots, m .
$$

Then for any function $g \in L^{\infty}\left(\mathscr{H}_{m, q} ; \mathbf{R}\right)$,

$$
f(\sigma):=g(F(\sigma))=g(\eta) \in L^{\infty}(\Sigma ; \mathbf{R}) .
$$

Recall that from the definition of the generator $M_{N}$ of $\left\{\sigma_{t}\right\}_{t \geqslant 0}$ we have that for all test functions $f \in L^{\infty}(\Sigma ; \mathbf{R})$,

$$
\frac{\mathrm{d}}{\mathrm{d} t} E f(\sigma)=E \sum_{x, y \in \mathscr{L}} c(x, y, \sigma)\left[f\left(\sigma^{(x, y)}\right)-f(\sigma)\right]=E \sum_{k, l \in \mathscr{L}_{\mathrm{c}}} \sum_{x \in D_{k}, y \in D_{l}} c(x, y, \sigma)\left[f\left(\sigma^{(x, y)}\right)-f(\sigma)\right] .
$$

However a straightforward calculation yields for $x \in D_{k}, y \in D_{l}$,

$$
F\left(\sigma^{(x, y)}\right)=F(\sigma)+\sigma(y)(1-\sigma(x))\left(\delta_{k}-\delta_{l}\right)+\sigma(x)(1-\sigma(y))\left(\delta_{l}-\delta_{k}\right) .
$$

Thus, for the test functions $f$ defined in (A.8) and $x \in D_{k}, y \in D_{l}$ we have:

$$
f\left(\sigma^{(x, y)}\right)=\sigma(y)(1-\sigma(x)) g\left(\eta+\delta_{k}-\delta_{l}\right)+\sigma(x)(1-\sigma(y)) g\left(\eta+\delta_{l}-\delta_{k}\right) .
$$


Substituting in (A.9) we obtain:

$$
\begin{aligned}
\frac{\mathrm{d}}{\mathrm{d} t} E g(\eta)= & E \sum_{k, l \in \mathscr{L}_{\mathrm{c}}} \sum_{x \in D_{k}, y \in D_{l}} c(x, y, \sigma)\left[\sigma(y)(1-\sigma(x)) g\left(\eta+\delta_{k}-\delta_{l}\right)\right. \\
& \left.+\sigma(x)(1-\sigma(y)) g\left(\eta+\delta_{l}-\delta_{k}\right)-g(\eta)\right]
\end{aligned}
$$

where the rate $c(x, y, \sigma)$ is given by (A.7), when $x, y$ are nearest neighbors (it is equal to zero otherwise) and $x \in D_{k}, y \in D_{l}$ :

$$
c(x, y, \sigma)=c_{0} \sigma(x)(1-\sigma(y)) \exp \left[-\beta\left(U_{0}+U(x)\right)\right]+c_{0} \sigma(y)(1-\sigma(x)) \exp \left[-\beta\left(U_{0}+U(y)\right)\right] .
$$

As in the case of adsorption/desorption we can replace $U(x)$ by $\bar{U}(k)$ (resp. $U(y)$ by $\bar{U}(l)$ ). If in addition we make the closure assumption that the particles are approximately independent inside each coarse cell $D_{k}$, we can replace $\sigma(x)$ by $q^{-1} \eta(k)$ (resp. $\sigma(y)$ by $q^{-1} \eta(l)$ ).

Then using the previous approximations in (A.10) we can define the new coarse-grained Markov process $\eta$ on $\mathscr{H}_{m, q}$, satisfying for any $g \in L^{\infty}\left(\mathscr{H}_{m, q} ; \mathbf{R}\right)$,

$$
\frac{\mathrm{d}}{\mathrm{d} t} E g(\eta)=E \sum_{k, l \in \mathscr{L}_{\mathrm{c}}} \bar{c}(k \mapsto l, \eta)\left[g\left(\eta+\delta_{l}-\delta_{k}\right)-g(\eta)\right] .
$$

The new rate $\bar{c}(k \mapsto l, \eta)$ describes the migration of a particle from the coarse cell $D_{k}$ to cell $D_{l}$. It is is defined using (A.11) as well as a time rescaling, as:

$$
\bar{c}(k \mapsto l, \eta)=\frac{1}{q} \eta(k)(q-\eta(l)) c_{0} \exp \left[-\beta\left(U_{0}+\bar{U}(k)\right)\right],
$$

if $k, l$ are nearest neighbors and $\bar{c}(k \mapsto l, \eta)=0$ otherwise. The corresponding coarse-grained generator is according to (A.12):

$$
M_{\mathrm{c}} g(\eta):=\sum_{k, l \in \mathscr{L}_{\mathrm{c}}} \bar{c}(k \mapsto l, \eta)\left[g\left(\eta+\delta_{l}-\delta_{k}\right)-g(\eta)\right] .
$$

\section{References}

[1] G. Beylkin, On the representation of operators in bases of compactly supported wavelets, SIAM J. Numer. Anal. 29 (1992) 1716.

[2] G. Beylkin, R. Coifman, V. Rokhlin, Fast wavelet transforms and numerical algorithms. I, Comm. Pure Appl. Math. 44 (1991) 141.

[3] C. Boucher, R.S. Ellis, B. Turkington, Derivation of maximum entropy principles in two-dimensional turbulence via large deviations, J. Stat. Phys. 98 (2000) 1235.

[4] A. De Masi, E. Orlandi, E. Presutti, L. Triolo, Glauber evolution with Kač potentials: I. Mesoscopic and macroscopic limits, interface dynamics, Nonlinearity 7 (1994) 633.

[5] P. Dupuis, R.S. Ellis, A Weak Convergence Approach to the Theory of Large Deviations, Wiley, New York, 1997.

[6] T. Eisele, R.S. Ellis, Symmetry breaking and random waves for magnetic systems on a circle, Z. Wahrsch. Verw. Gebiete 63 (1983) 297.

[7] G. Giacomin, J.L. Lebowitz, Phase segregation dynamics in particle systems with long range interactions.I. Macroscopic limits, J. Stat. Phys. 87 (1997) 37.

[8] G. Giacomin, J.L. Lebowitz, E. Presutti, Deterministic and stochastic hydrodynamic equations arising from simple microscopic model systems, in: Stochastic Partial Differential Equations: Six Perspectives, in: R. Carmona and B. Rozovskii (Eds.), Math. Surveys Monogr., vol. 64, Amer. Math. Soc, Providence, RI, 1999, p. 107.

[9] N. Goldenfeld, Lectures on Phase Transitions and the Renormalization Group, 85, Addison-Wesley, New York, 1992 , Chapter 9.

[10] M. Hildebrand, A.S. Mikhailov, Mesoscopic modeling in the kinetic theory of adsorbates, J. Phys. Chem. 100 (1996) 19089.

[11] M. Hildebrand, A.S. Mikhailov, G. Ertl, Nonequilibrium stationary microstructures in surface chemical reactions, Phys. Rev. E 58 (1998) 5483. 
[12] T.L. Hill, An Introduction to Statistical Thermodynamics, Dover, New York, 1986.

[13] D.J. Horntrop, M.A. Katsoulakis, D.G. Vlachos, Spectral methods for mesoscopic models in pattern formation, J. Comp. Phys. 173 (2001) 364.

[14] S. Jakubith, H.H. Rotermund, W. Engel, A. Von Oertzen, G. Ertl, Spatiotemporal concentration patterns in a surface reaction: propagating and standing waves, rotating spirals and turbulance, Phys. Rev. Lett. 65 (1990) 3016.

[15] L.P. Kadanoff, Scaling laws for Ising models near $T_{\mathrm{c}}$, Physics 2 (1966) 263.

[16] M.A. Katsoulakis, P.E. Souganidis, Stochastic Ising models and anisotropic front propagation, J. Stat. Phys. 87 (1997) 63.

[17] J.S. Langer, Theory of spinodal decomposition in alloys, Ann. Phys. 65 (1971) 53.

[18] T.M. Liggett, Stochastic interacting systems: contact, voter and exclusion processes, Grundlehren der Mathematischen Wissenschaften, vol. 324, Springer, Berlin, 1999.

[19] A.J. Majda, B. Khouider, Stochastic and mesoscopic models for tropical convection, Proc. Natl. Acad. Sci. USA 99 (2002) 1123.

[20] A. Milchev, D.W. Heermann, K. Binder, Monte Carlo simulation of the Cahn-Hilliard model of spinodal decomposition, Acta Metall. 36 (1988) 377.

[21] J.S. Reese, S. Raimondeau, D.G. Vlachos, Monte Carlo algorithms for complex surface reaction mechanisms: efficiency and accuracy, J. Comp. Phys. 173 (1) (1999) 302-321.

[22] A.N. Shiryaev, Probability, Graduate Texts in Mathematics, vol. 95, Springer, New York, 1996.

[23] D.G. Vlachos, The role of macroscopic transport phenomena in film microstructure during epitaxial growth, Appl. Phys. Lett. 74 (19) (1999) 2797.

[24] D.G. Vlachos, M.A. Katsoulakis, Derivation and validation of mesoscopic theories for diffusion-reaction of interacting molecules, Phys. Rev. Lett. 85 (2000) 3898. 